\title{
Fatty acid oxidation by the osteoblast is required for normal bone acquisition in a sex- and diet-dependent manner
}

\author{
Soohyun P. Kim, ${ }^{1}$ Zhu Li, ${ }^{1}$ Meredith L. Zoch, ${ }^{1}$ Julie L. Frey, ${ }^{1}$ Caitlyn E. Bowman, ${ }^{2}$ Priyanka Kushwaha, \\ Kathleen A. Ryan, ${ }^{1}$ Brian C. Goh, ${ }^{1}$ Susanna Scafidi, ${ }^{3}$ Julie E. Pickett, ${ }^{4}$ Marie-Claude Faugere, ${ }^{5}$ \\ Erin E. Kershaw, ${ }^{6}$ Daniel L. J. Thorek, ${ }^{4,7}$ Thomas L. Clemens, ${ }^{1,8}$ Michael J. Wolfgang, ${ }^{2}$ \\ and Ryan C. Riddle ${ }^{1,8}$ \\ 'Department of Orthopaedic Surgery, ${ }^{2}$ Department of Biological Chemistry, and ${ }^{3}$ Department of Anesthesiology and \\ Critical Care Medicine, Johns Hopkins University School of Medicine, Baltimore, Maryland, USA. ${ }^{4}$ Division of Nuclear \\ Medicine and Molecular Imaging, Department of Radiology and Radiological Science, Johns Hopkins University School of \\ Medicine, Baltimore, Maryland, USA. ${ }^{5}$ Division of Nephrology, University of Kentucky, Lexington, Kentucky, USA. ${ }^{6}$ Division \\ of Endocrinology, Department of Medicine, University of Pittsburgh, Pittsburgh, Pennsylvania, USA. ${ }^{7}$ Cancer Molecular \\ and Functional Imaging Program, Department of Oncology, Sidney Kimmel Comprehensive Cancer Center, Johns Hopkins \\ University School of Medicine, Baltimore, Maryland, USA. ${ }^{8}$ Baltimore Veterans Administration Medical Center, Baltimore, \\ Maryland, USA.
}

\begin{abstract}
Postnatal bone formation is influenced by nutritional status and compromised by disturbances in metabolism. The oxidation of dietary lipids represents a critical source of ATP for many cells but has been poorly studied in the skeleton, where the prevailing view is that glucose is the primary energy source. Here, we examined fatty acid uptake by bone and probed the requirement for fatty acid catabolism during bone formation by specifically disrupting the expression of carnitine palmitoyltransferase 2 (Cpt2), an obligate enzyme in fatty acid oxidation, in osteoblasts and osteocytes. Radiotracer studies demonstrated that the skeleton accumulates a significant fraction of postprandial fatty acids, which was equal to or in excess of that acquired by skeletal muscle or adipose tissue. Female, but not male, Cpt2 mutant mice exhibited significant impairments in postnatal bone acquisition, potentially due to an inability of osteoblasts to modify fuel selection. Intriguingly, suppression of fatty acid utilization by osteoblasts and osteocytes also resulted in the development of dyslipidemia and diet-dependent modifications in body composition. Taken together, these studies demonstrate a requirement for fatty acid oxidation during bone accrual and suggest a role for the skeleton in lipid homeostasis.
\end{abstract}

Authorship note: SPK and ZL contributed equally to this work.

Conflict of interest: The authors have declared that no conflict of interest exists.

Submitted: January 9, 2017 Accepted: July 19, 2017 Published: August 17, 2017

\section{Reference information:} JCI Insight. 2017;2(16):e92704 https://doi.org/10.1172/jici. insight.92704.

\section{Introduction}

The formation and maintenance of the vertebrate skeleton are energy-intensive processes that impact global energy expenditure and are influenced by metabolic hormones like insulin, leptin, and adiponectin (1-5). In states of extreme malnourishment, bone growth ceases and a reduction in bone mass is associated with increased fracture risk (6-8). In addition, the metabolic disturbances associated with diabetes are accompanied by abnormal bone mineral density and increased fracture risk (9-11).

To date, the vast majority of studies on the intermediary metabolism of osteoblasts have focused on the utilization of glucose. This concept stems from early studies that illustrated cultured osteoblasts metabolize glucose to lactate (12-14) and that osteoanabolic hormones and growth factors stimulate glucose consumption $(15,16)$. Recent studies have confirmed these findings using more refined genetic and molecular techniques and elaborated on mechanisms of glucose acquisition and utilization (17-20). For instance, transport by glucose transporter-1 (Glut1) instructs the commitment of early progenitors to the osteoblast lineage by inhibiting the activation of AMP-activated protein kinase, a sensor of cellular energy status, and thereby stabilizing the master osteogenic transcription factor Runx2 (21). Likewise, expression of Glut4 allows osteoblasts to contribute to the clearance of glucose from the circulation (22). 
A
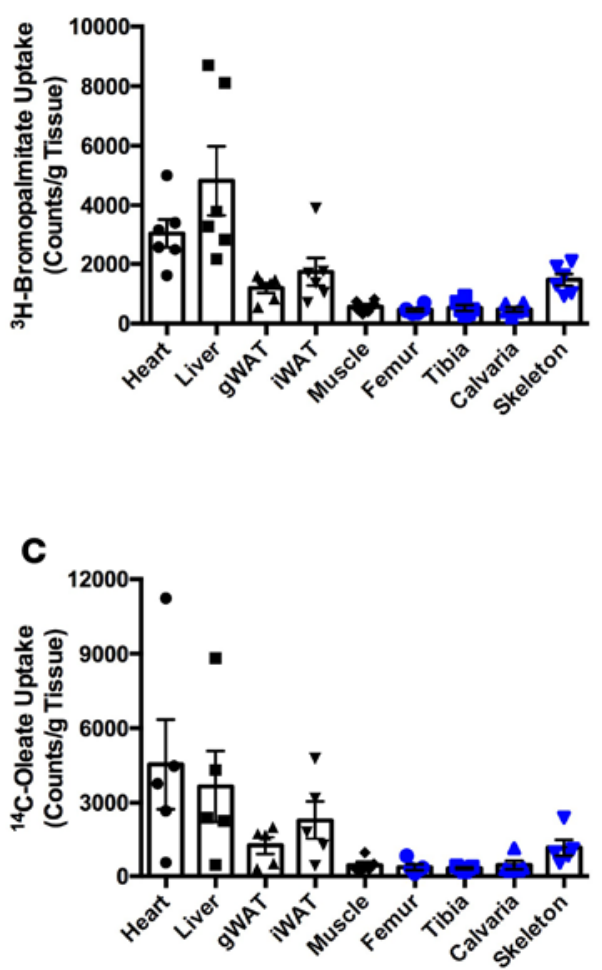

B
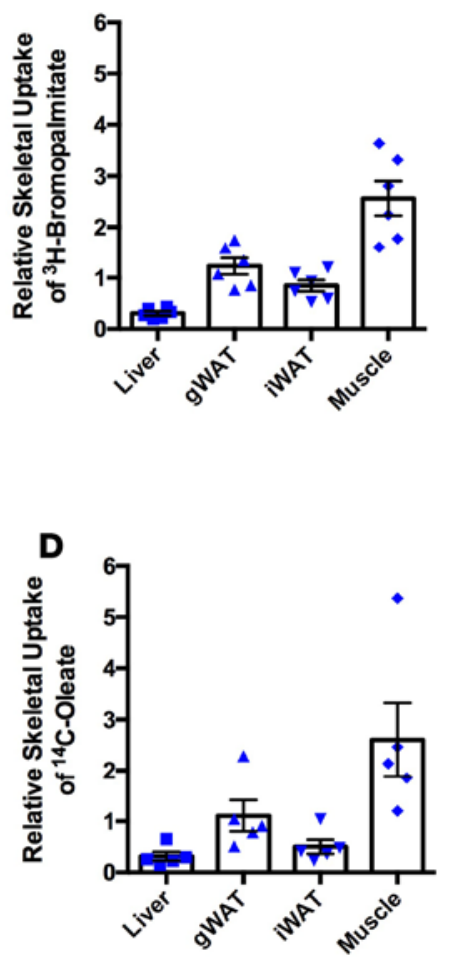

E

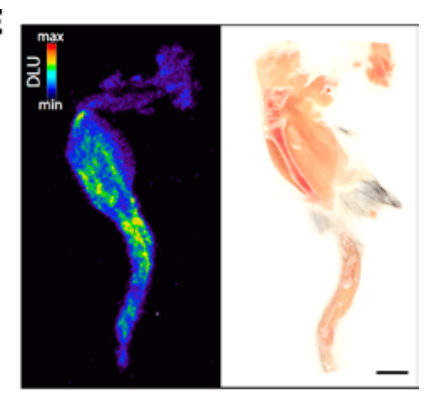

Figure 1. Bone takes up a substantial fraction of postprandial lipid. (A and C) Biodistribution of $\left[{ }^{3} \mathrm{H}\right]$-bromopalmitate $(\mathbf{A})$ and $\left[{ }^{14} \mathbf{C}\right]$-oleate $(\mathbf{C})$ in 12 -week-old C57BI/6 mice ( $n=5-6$ mice). Uptake (cpm) is normalized for tissue weight. Skeleton represents the combined uptake by femur, tibia, and calvaria. (B and D) Levels of $\left[{ }^{3} \mathrm{H}\right]$-bromopalmitate $(\mathbf{B})$ and $\left[{ }^{14} \mathrm{C}\right]$-oleate (D) uptake in the skeleton relative to the indicated tissue. (E) Autoradiographic analysis of ${ }^{125}$-BMIPP uptake in the tibia, with whole mount tissue section on right (Representative of $n=4$ mice). (F) Comparison of [ $\left.{ }^{3} \mathrm{H}\right]$-acetate incorporation into tissue lipids in gonadal white adipose and the femur ( $n=6-7$ mice). All data are represented by mean \pm SEM. ${ }^{*} P<0.05$ by unpaired, two-tailed Student's $t$ test.

Many cells also depend on fatty acids to generate ATP, which — by weight — yields more energy than carbohydrates. Initial studies suggested that fatty acid metabolism contributes as little as $40 \%$ of the energy yield that cultured osteoblasts derive from the metabolism of glucose (23). However, our previous work demonstrated that fatty acid $\beta$-oxidation increases dramatically as osteoblasts mature in vitro and that anabolic Wnt signaling through the low-density lipoprotein-related receptor-5 promotes fatty acid oxidation (24). Similarly, histological and radiotracer studies indicate that bone takes up a significant fraction of postprandial lipoproteins (25), while in vitro studies suggest that high- and low-density lipoproteins are sufficient to maintain osteoblast proliferation under serum-free conditions (26).

Here, we investigated the requirement for fatty acid oxidation during postnatal bone acquisition by disrupting the expression of carnitine palmitoyltransferase-2 (Cpt2), an obligate enzyme in mitochondrial fatty acid $\beta$-oxidation, in osteoblasts and osteocytes. The translocation of long-chain fatty acids into the mitochondrial matrix for $\beta$-oxidation requires the activity of 2 carnitine acyl-transferases. Cpt1, which transfers fatty acyl groups from CoA to carnitine, is associated with the outer mitochondrial membrane and is the rate-limiting enzyme in fatty acid $\beta$-oxidation. However, this enzyme is encoded by 3 isoforms (Cpt-1a, Cpt-1b, and Cpt-1c) with overlapping tissue expression patterns (27-31) and the potential for functional compensation among isoforms in gene loss of function studies (32). Cpt2, which reverses the reaction catalyzed by $\mathrm{Cpt} 1$ after the shuttling of acylcarnitines by specific transporters into the mitochondrial matrix, is associated with the inner mitochondrial membrane and encoded by a single gene (30).

Here, we report that the loss of Cpt 2 function impairs postnatal bone acquisition and osteoblast differentiation. These defects are particularly severe in female mice and osteoblasts exposed to estrogen, which exhibit an impaired ability to enhance glucose utilization in the face of impaired fatty acid metabolism. Intriguingly, deficiencies in fatty acid utilization by osteoblasts and osteocytes are also sufficient to alter whole body lipid homeostasis and, in the context of high fat-diet feeding, result in increased adiposity, presumably due to the reallocation of energy sources. 


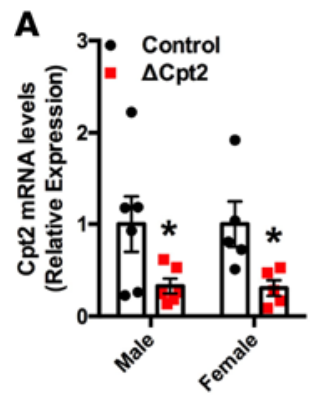

$\mathbf{E}$
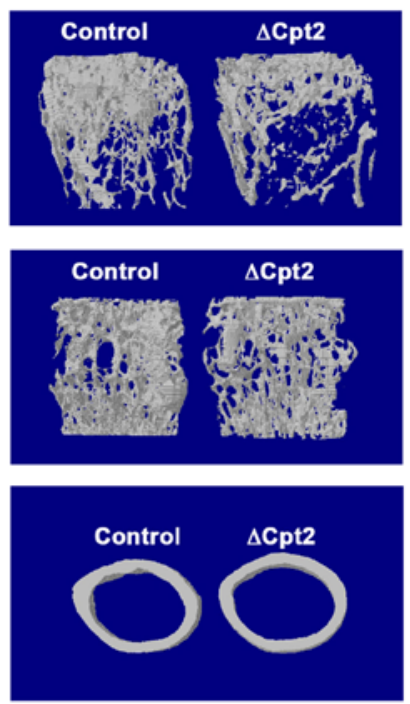

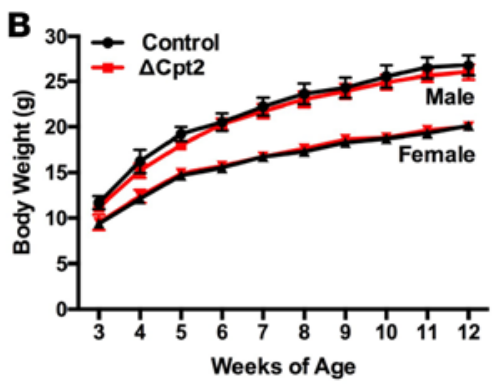

F
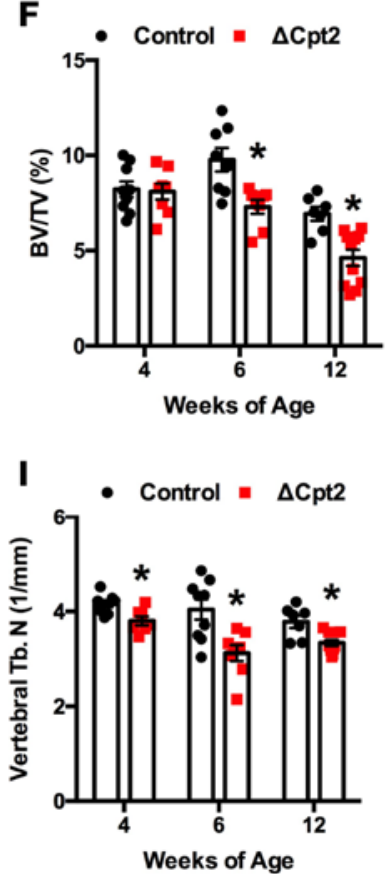
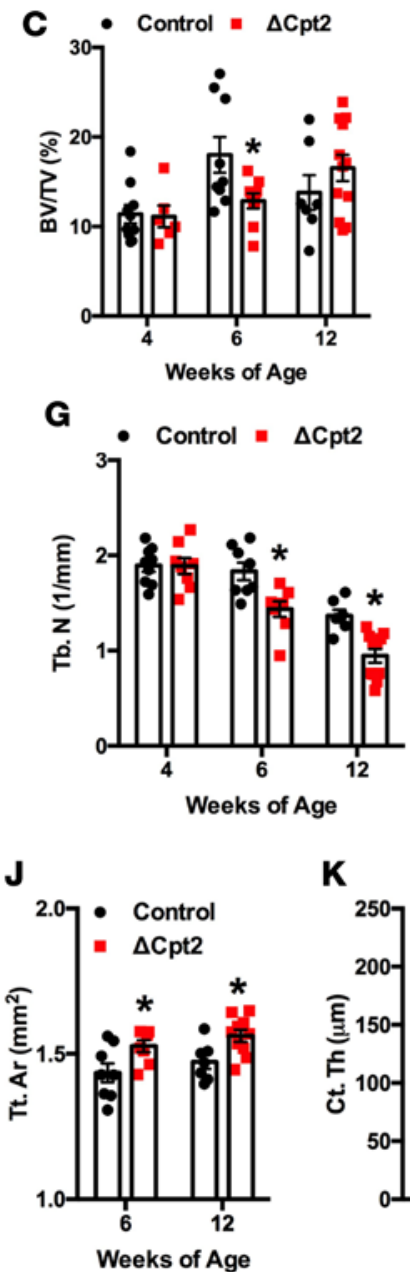

$\mathbf{K}$
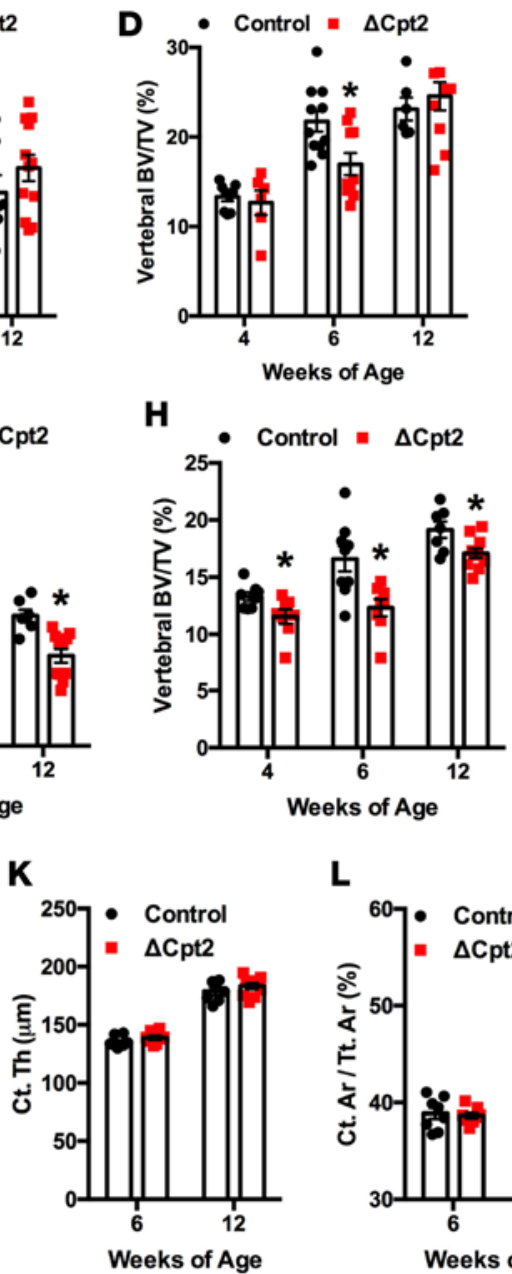

$\mathbf{L}$

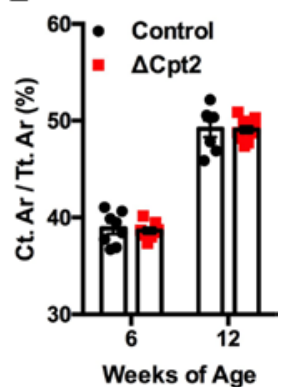

Figure 2. Fatty acid oxidation is required for the maintenance of normal bone structure in female mice. (A) qPCR analysis of Cpt2 mRNA levels in the femur of 6-week-old control and $\Delta$ Cpt2 mice $(n=5-7$ mice). (B) Body weight was assessed weekly from 3-12 weeks of age ( $n=7-11$ mice). (C and $\mathbf{D})$ Quantification of trabecular bone volume in the distal femur (C) and L5 vertebrae (D) of male control and $\Delta$ Cpt2 mice $(n=6-12$ mice). (E) Representative computer renderings of bone structure in the distal femur (top), L5 vertebrae (middle), and femoral mid-diaphysis (bottom) of 6-week-old female control and $\Delta$ Cpt2 mice. (F-I) Quantification of trabecular bone volume per tissue volume ( $F$ and $\mathbf{H}, \mathrm{BV} / \mathrm{TV}$ ), trabecular number ( $\mathbf{G}$ and $\mathbf{I}$, Tb.N) in the distal femur ( $\mathbf{F}$ and $\mathbf{G}$ ) and $\mathbf{L} 5$ vertebrae ( $\mathbf{H}$ and $\mathbf{I})$ of female control and $\Delta$ Cpt2 mice ( $n=7-11$ mice). (J-L) Quantification of cortical tissue area (J, Tt.Ar), cortical thickness (K, Ct.Th), and cortical area per tissue area (L, Ct.Ar/Tt.Ar) at the mid-diaphysis of female control and $\Delta$ Cpt2 mice $(n=7-10$ mice). All data are represented by mean \pm SEM. ${ }^{*} P<0.05$ by unpaired, two-tailed Student's $t$ test.

\section{Results}

Skeletal accumulation of fatty acids. As a first step in characterizing fatty acid uptake by the skeleton and to determine the relative contribution of bone to whole-body fatty acid metabolism, we administered radiolabeled fatty acid tracers by gavage and performed biodistribution studies. As expected, the uptake of ${ }^{3} \mathrm{H}$-bromopalmitate, a non- $\beta$-oxidizable analog of the most common fatty acids in animals, and ${ }^{14} \mathrm{C}$-oleate, a monounsaturated fatty acid, was greatest in the heart and liver (Figure $1, \mathrm{~A}$ and $\mathrm{C}$ ). Significant uptake of both tracers was also evident in the femur, tibia, and calvaria. When normalized for tissue weight, uptake in the selected bones was approximately $80 \%$ of that taken up by the quadriceps femoris muscle, $40 \%$ of that taken up by the gonadal fat pad, and $10 \%$ of that taken up by the liver. When the levels of uptake in the femur, tibia, and calvaria were combined (skeleton), they exceeded those evident in gonadal fat and the quadriceps femoris muscle (Figure 1, B and D), implying that bone plays a significant role in the clearance of plasma fatty acids.

To localize fatty acid uptake in bone, we administered the long-chain fatty acid tracer ${ }^{125}$ I-iodine-15-(p-iodophenyl)-3(R,S)-methylpentadecanoic acid (BMIPP) $(33,34)$ and performed autoradiography analyses on the tibia. BMIPP uptake was primarily localized to the cortical bone envelope and the 
Table 1.

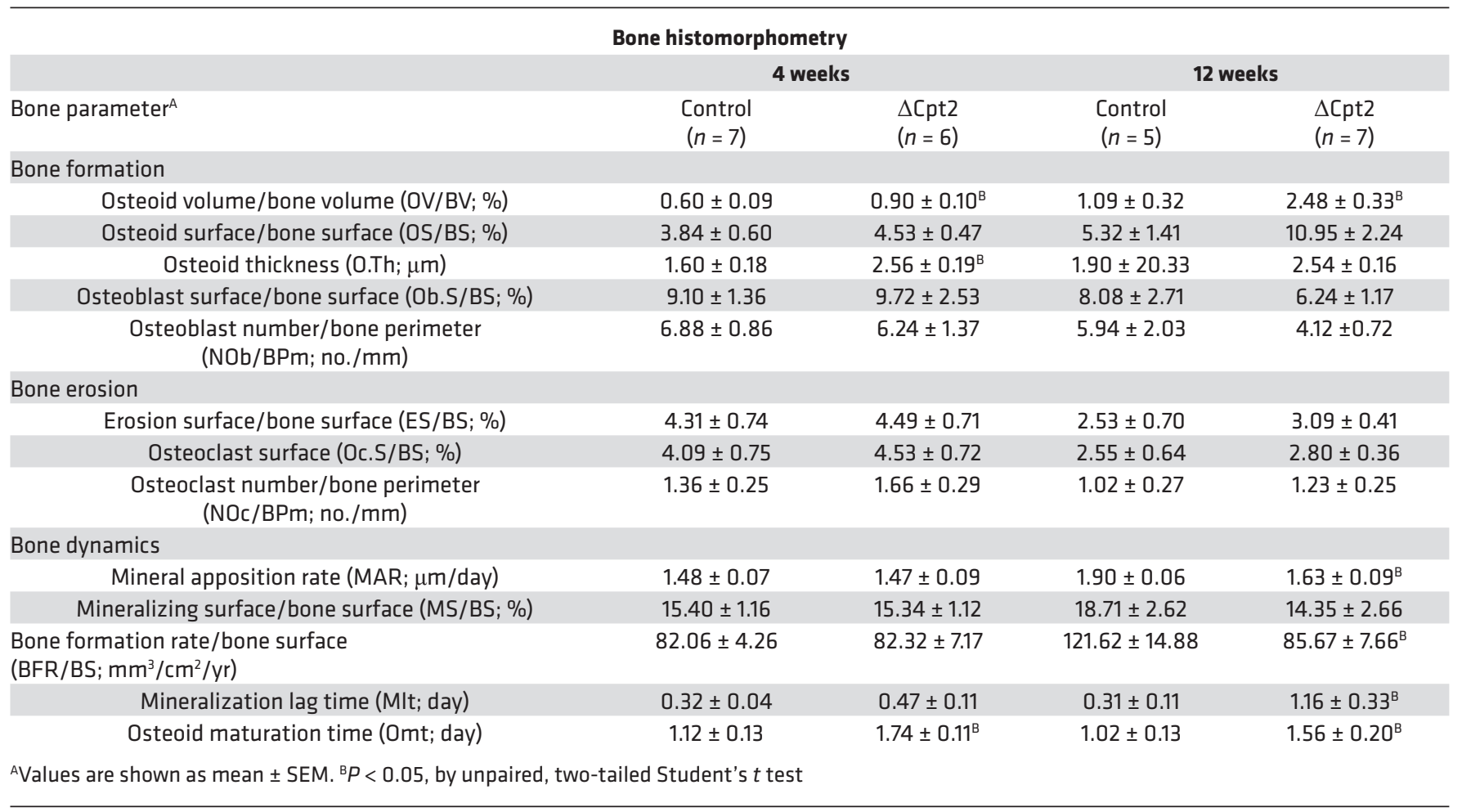

trabecular bone compartment under the growth plate (Figure 1E). Assessment of de novo fatty acid synthesis, indexed by the incorporation of ${ }^{3} \mathrm{H}$-acetate into tissue lipids, indicated that bone is unlikely to contribute significantly to fatty acid anabolism, as the level of acetate incorporation in the femurs of male mice was less than $30 \%$ of those evident in white adipose tissue (Figure $1 \mathrm{~F}$ ). Since bone does not store or synthesize a significant amount of fat, acquired fatty acids are likely to be oxidized for energy production.

Loss of Cpt2 function in osteoblasts impairs bone acquisition in female mice. To characterize the requirement for fatty acid $\beta$-oxidation during bone formation, we generated mice in which the $C p t 2$ gene that encodes Cpt2 was selectively disrupted in osteoblasts and osteocytes. Cpt2 $2^{\text {lox/lox }}$ mice in which exon 4 is flanked by loxP sites $(35,36)$ were crossed with Oc-Cre mice (37) to generate osteoblast-specific mutants (Oc-Cre ${ }^{\mathrm{TG} /+}$; $\mathrm{Cpt} 2^{\text {lox } / \mathrm{lox}}$, hereafter referred to as $\left.\Delta \mathrm{Cpt} 2\right)$ and control littermates $\left(\mathrm{Cpt} 2^{\mathrm{lox} / \mathrm{lox}}\right)$. Both male and female $\Delta \mathrm{Cpt} 2$ mice were born at the expected Mendelian frequency, exhibited 70\% reductions in Cpt2 mRNA levels in skeletal tissue (Figure 2A) with normal expression levels in other metabolic tissues (Supplemental Figure 1; supplemental material available online with this article; https://doi.org/10.1172/jci.insight.92704DS1), and maintained normal body weights (Figure 2B).

Male $\Delta$ Cpt2 mice exhibited only transient deficits in skeletal architecture. MicroCT analyses revealed normal bone structure in the mutant mice at 4 weeks of age, but significant reductions in trabecular bone volume (Figure 2, C and D) - in association with modest declines in both trabecular number and thickness (data not shown) - were evident in both the distal femur and L5 vertebrae at 6 weeks of age. With advancing age (12 weeks), this phenotype completely resolved. By contrast, female $\Delta \mathrm{Cpt} 2$ mice exhibited persistent deficits in bone volume. Female mutants failed to attain the peak in femoral trabecular bone volume evident at 6 weeks of age in control littermates (Figure 2, E-G) and maintained a low bone volume phenotype through 12 weeks of age. Similar reductions in trabecular bone volume and trabecular number were also evident in the spine, where the phenotype was apparent as early as 4 week of age (Figure $2, \mathrm{H}$ and I). Female $\Delta \mathrm{Cpt} 2$ mutants also exhibited significant increases in cortical bone tissue area in the femur without changes in cortical bone thickness (Figure 2, J-L), which raises the possibility that skeletal architecture has adapted to maintain bone strength in the face of inferior bone quality. 
To examine the cellular basis for the reduced bone volume in female $\Delta \mathrm{Cpt} 2$ mice, we next performed static and dynamic histomorphometric analyses in the trabecular bone compartment of the distal femur at both 4 and 12 weeks of age (Table 1). At 4 weeks of age, the numbers of osteoblasts per bone perimeter (Ob.N/BPM) and dynamic indices of bone formation (mineral apposition rate [MAR], mineralizing surface/bone surface [MS/BS], and bone formation rate per bone surface [BFR/BS]) were equivalent in control and $\Delta \mathrm{Cpt} 2$ mice. However, significant increases in the osteoid thickness (O.Th), osteoid volume per bone volume (OV/BV), and the osteoid maturation time (Omt) in $\Delta \mathrm{Cpt} 2$ mice relative to controls suggested the emergence of a mineralization defect. Consistent with this idea, the increase in osteoid volume and Omt persisted through 12 weeks of age and was accompanied by significant decreases in the MAR and $\mathrm{BFR} / \mathrm{BS}$, along with increases in the mineralization lag time (Mlt), presumably due to the reductions in catabolic metabolism and the development of an energy stress response (Figure 3, M and N). The numbers of osteoclasts per tissue perimeter (Oc.N/BPM) were not affected at either time point.

To determine if osteoblasts rely primarily on exogenous fatty acids or utilize intracellular stores, we used the same genetic strategy to disrupt the Pnpla2 gene that encodes adipose triglyceride lipase (Atgl), the first and rate-limiting enzyme in the hydrolysis of intracellular triglycerides (38), in osteoblasts lacking Atgl $(\triangle \mathrm{Atgl})$. The loss of Atgl function did not affect body weight or trabecular bone acquisition (Supplemental Figure 2). Likewise, oxidation of fatty acids and matrix mineralization proceeded normally in in vitro cultures of $\Delta \mathrm{Atgl}$ osteoblasts. Therefore, oxidation of acquired fatty acids by the osteoblast is required to fuel normal bone acquisition and mineralization in female and, to lesser extent, male mice.

Estrogen exacerbates defects in osteoblast differentiation associated with Cpt2 loss of function. To further examine the requirement for fatty acid oxidation during bone formation, we assessed osteoblast performance in vitro. Calvarial osteoblasts were isolated from $\mathrm{Cpt} 2^{\text {lox}} / \mathrm{lox}$ mice and were infected with adenoviral constructs directing the expression of $C r e$ recombinase ( $\triangle \mathrm{Cpt}$ ) or green fluorescent protein (control). $\Delta \mathrm{Cpt} 2$ cultures exhibited a more than $97 \%$ reduction in the levels of Cpt 2 mRNA relative to control cultures (Figure $3 \mathrm{~A}$ ), as well as a $60 \%$ reduction in the ability to fully oxidize ${ }^{14} \mathrm{C}$-labeled oleic acid to ${ }^{14} \mathrm{CO}_{2}$ (Figure $3 \mathrm{~B}$ ) - a $58 \%$ reduction in the oxygen consumption rate when cultured in the presence of oleate (Figure $3 \mathrm{C}$ ) - and the accumulation of several acylcarnitine species (Supplemental Table 2). The remaining capacity of $\Delta \mathrm{Cpt} 2$ cultures to oxidize oleic acid is likely due to either peroxisomal fatty acid oxidation or $\omega$-oxidation. Consistent with this idea, the levels of some hydroxylated acyl-carnitines were reduced in $\Delta$ Cpt 2 osteoblast cultures relative to controls (Supplemental Table 2).

Loss of Cpt2 function resulted in a mild impairment in osteoblast differentiation in vitro that was marked by significant decreases in the expression of the osteogenic markers Runx2, Sp7 (Osterix), and Bglap2 (Osteocalcin) (Figure 3D), as well as reduced staining for alkaline phosphatase activity and a $20 \%$ decrease in matrix mineralization (Figure 3, E and F). The expression levels of genes associated with osteoclast development were not affected (data not shown). In accordance with the more dramatic defect in skeletal architecture evident in female Cpt2 mutants (Figure 2), the addition of $10 \mathrm{nM} 17 \beta$-estradiol (E2) to cultures resulted in a more substantial impairment in the maturation of $\Delta$ Cpt 2 osteoblasts (Figure 3, D-F). E2 treatment of control osteoblasts produced the expected osteogenic effect, but the expression levels of Runx2, Osterix, and Bglap2 and matrix mineralization (38\%) were reduced to a greater degree in E2-treated $\Delta \mathrm{Cpt} 2$ osteoblasts when compared with vehicle-treated, mutant cultures. Likewise, the expression level of Colla1, which was not affected by Cpt2 ablation alone, was significantly downregulated after E2 treatment.

We speculated that the more severe defect in in vitro maturation induced by E2 treatment of $\Delta \mathrm{Cpt} 2$ osteoblast might be due to an inability to adjust fuel selection, since E2 favors fatty acid utilization in a number of tissues (39-42). To test this idea, control and $\Delta \mathrm{Cpt} 2$ osteoblast cultured in the presence or absence of E2 were labeled with 2-deoxy-D-[ $\left.{ }^{3} \mathrm{H}\right]$-glucose to assess glucose uptake. When compared with controls, $\Delta \mathrm{Cpt} 2$ osteoblasts exhibited a $17 \%$ increase in 2-deoxy-D- $\left[{ }^{3} \mathrm{H}\right]$-glucose acquisition (Figure $3 \mathrm{G}$ ), suggestive of an adjustment in cellular metabolism to maintain ATP levels. Likewise, $\Delta \mathrm{Cpt} 2$ osteoblasts generated more lactate (Figure $3 \mathrm{H}$ ), exhibited an increase in the expression of genes encoding lactate dehydrogenase and Glut1 (Figure 3I), and displayed an increase in the extracellular acidification rate (Figure 3K) without a change in oxygen consumption rate (Figure 3J) when cultured in the presence of $2.5 \mathrm{mM}$ glucose. This shift toward glycolytic metabolism was completely inhibited in $\triangle \mathrm{Cpt} 2$ osteoblast cultures treated with E2. The acquisition of glutamine, another potential energy source for osteoblasts (43), was not affected by Cpt2 deficiency and was mildly suppressed by E2 treatment (Figure $3 \mathrm{~L}$ ). As a result, $\Delta \mathrm{Cpt} 2$ osteoblast cultures 
A
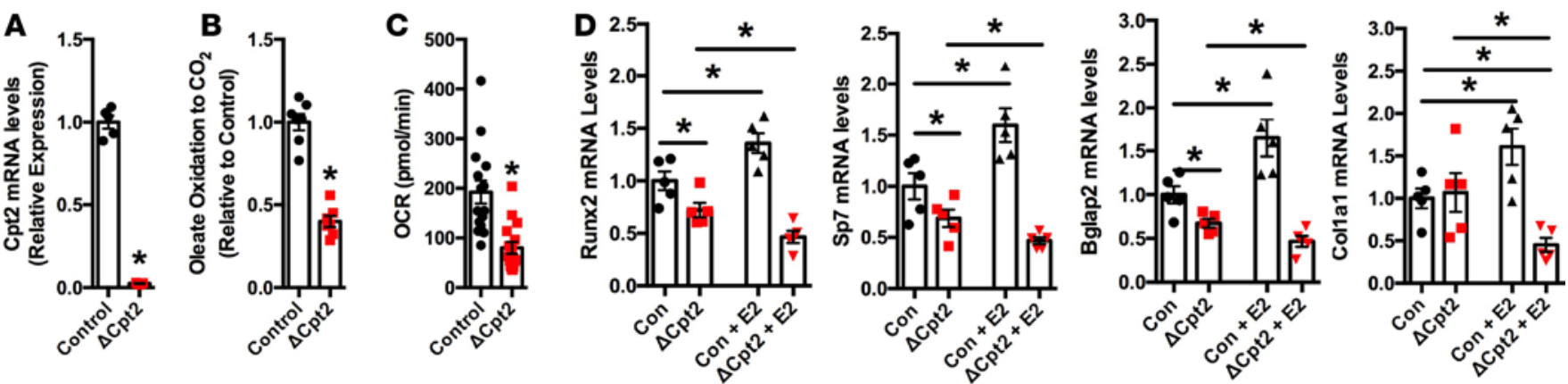

E
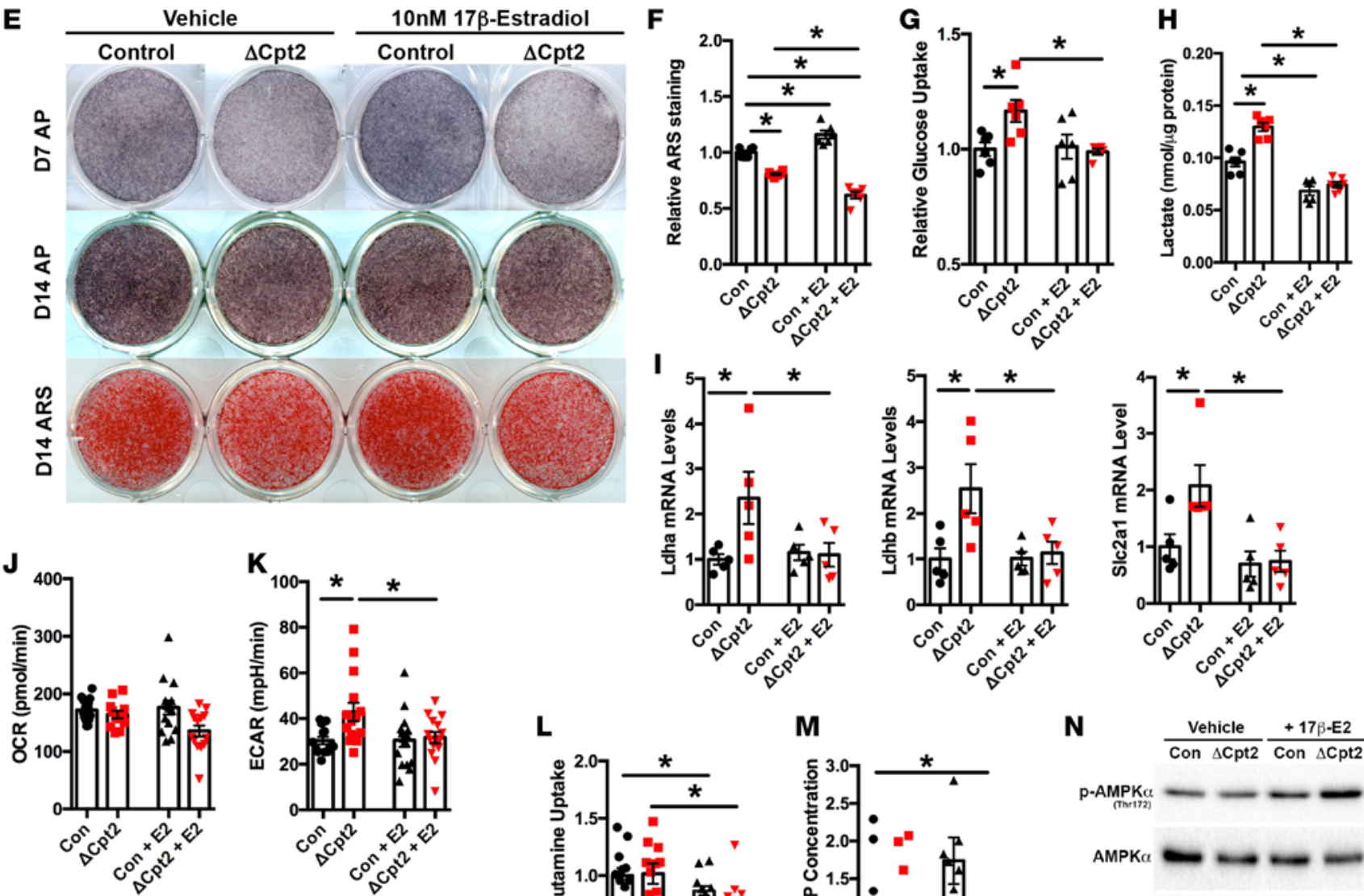

L

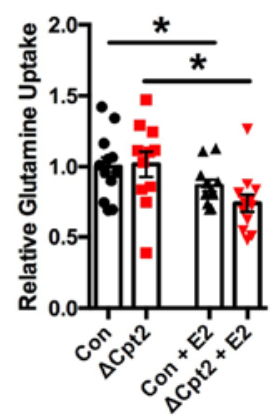

M

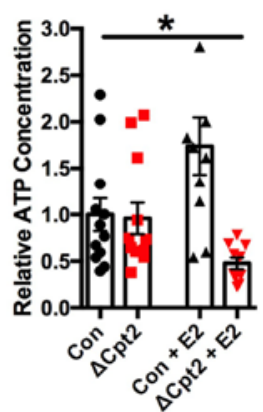

$\mathbf{N}$

$\frac{\text { Vehicle }}{\text { Con } \Delta \mathrm{Cpt} 2} \frac{+17 \beta-\mathrm{E} 2}{\operatorname{Con} \Delta \mathrm{Cpt} 2}$

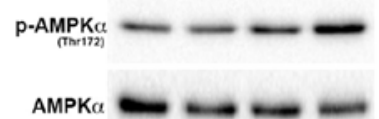

P-AMPKB1 $\cdots$

AMPK $\beta 1 / 2 \div-$

Figure 3. In vitro differentiation of osteoblasts is impaired following the ablation of Cpt2 expression. (A) qPCR analysis of Cpt2 mRNA levels in primary osteoblasts after infection with adenoviral constructs encoding GFP (control) or Cre-recombinase $(\Delta C p t 2)(n=6)$. (B) Relative levels of $\left[{ }^{14} \mathrm{C}\right]$-oleate oxidation to ${ }^{14} \mathrm{CO}_{2}(n=7)$. (C) Oxygen consumption rate $(\mathrm{OCR})$ in control and $\Delta$ Cpt2 osteoblasts in the presence of $200 \mu \mathrm{M}$ oleate $(n=16)$. (D) qPCR analysis of genes associated with osteoblastic differentiation in control and $\Delta$ Cpt2 osteoblasts cultured in the presence or absence of 10 $\mathrm{nM} 17 \beta$-estradiol (E2) $(n=5)$. (E) Staining for alkaline phosphatase activity (AP) on days 7 and 14 of in vitro differentiation and calcium deposition by alizarin red (ARS) on day 14 in cultures of control and $\Delta$ Cpt2 osteoblasts. (F) Quantification of relative ARS levels $(n=6)$. (G) Relative levels of 2-deoxy-D-[ $\left.{ }^{3} \mathrm{H}\right]$-glucose uptake in control and $\Delta$ Cpt2 osteoblasts cultured in the presence or absence of E2 $(n=6)$. (H) Cellular lactate levels in control and $\Delta$ Cpt2 osteoblasts $(n=5)$. (I) qPCR analysis of genes associated with glucose metabolism in control and $\Delta$ Cpt2 osteoblasts cultured in the presence or absence of E2 $(n=5)$. ( J and K) OCR (J) and extracellular acidification rate (ECAR, K) were assessed in osteoblasts cultured in the presence of $2.5 \mathrm{mM}$ glucose $(n=15-16)$. (L) Relative levels of [ $\left.{ }^{14} \mathrm{C}\right]$-glutamine uptake $(n=11-12)$. (M) Cellular ATP in control and $\Delta$ Cpt2 osteoblasts $(n=10-12)$. (N) Immunoblots showing AMPK phosphorylation levels (representative of $n=4$ ). All data are represented by mean \pm SEM. ${ }^{*} P<0.05$ by unpaired, twotailed Student's $t$ test or ANOVA. 
treated with E2 exhibited a reduction in cellular ATP levels (Figure 3M) and the development of an energy stress response marked by increased levels of AMPK $\alpha$ phosphorylation (Figure $3 \mathrm{~N}$ ). Taken together, these in vitro data reveal the importance of fatty acid oxidation during osteoblast differentiation and suggest that the impaired acquisition of peak bone mass in female $\Delta \mathrm{Cpt} 2$ mutants may be due to a compromised ability to adjust fuel selection.

Cpt2 deficiency alters body composition and glucose uptake by the skeleton in male mice. To determine the significance of increased glucose uptake by $\Delta \mathrm{Cpt} 2$ osteoblasts cultured in vitro, we administered the glucose analog ${ }^{18} \mathrm{~F}$-fluorodeoxyglucose $\left(\left[{ }^{18} \mathrm{~F}\right]\right.$-FDG) to cohorts of male and female control and $\Delta \mathrm{Cpt} 2$ mice and performed biodistribution studies (17). Consistent with our in vitro findings, $\left[{ }^{18} \mathrm{~F}\right]-\mathrm{FDG}$ uptake was increased in the femurs of male $\Delta \mathrm{Cpt} 2$ mice relative to littermate controls (Figure $4 \mathrm{~A}$ ). Similarly, the expression levels of Ldha, Ldhb, and Scl2a1 (encoding Glut1) were significantly increased in mRNA samples isolated from the femoral cortex, which is enriched in osteoblasts and osteocytes, from male $\Delta \mathrm{Cpt} 2$ mice (Figure $4 \mathrm{C}) .\left[{ }^{18} \mathrm{~F}\right]-\mathrm{FDG}$ uptake was not increased in the femur of female Cpt2 mutants (Figure 4B), nor was the expression of lactate dehydrogenase or Glut1 increased (Figure 4C), which further confirms the sexual dimorphism in the ability of osteoblasts to adjust fuel utilization.

The reduced uptake of $\left[{ }^{18} \mathrm{~F}\right]-\mathrm{FDG}$ in the inguinal white adipose tissue (iWAT) of male Cpt 2 mutants (Figure 4A) led us to question whether the loss of Cpt2 function in osteoblasts and osteocytes influenced body composition and whole body metabolism. Quantitative assessment of body composition by NMR and necropsy (Figure 4, D-G) revealed a reduction in fat mass in male, but not female, $\Delta \mathrm{Cpt} 2$ mice relative to control littermates. Additionally, histomorphometric analyses of the gonadal fat pad (Figure 4, H-J) demonstrated that adipocyte size was reduced in male mutants, which suggests that the increased utilization of glucose by Cpt2-deficient osteoblast in male mice might lead to reduced storage in adipose tissue. Liver morphology was not affected in $\Delta \mathrm{Cpt} 2$ mice (Figure $4 \mathrm{~K}$ ).

Despite the reduction in fat mass and reduced adipocyte size in male $\Delta \mathrm{Cpt} 2$ mice, random-fed glucose levels were equivalent to controls in both male and female $\Delta \mathrm{Cpt} 2$ mice (Figure $4 \mathrm{~L}$ ), and the mutant mice exhibited normal glucose dynamics in glucose-tolerance and insulin-tolerance tests (Supplemental Figure 3). However, both male and female mutants exhibited a dyslipidemia (Figure 4, M-Q). Serum free fatty acid levels were increased in both sexes relative to control littermates, while female mutants also had elevated triglyceride levels. Thus, the loss of Cpt2 function in osteoblasts and osteocytes is sufficient to disturb lipid homeostasis in both sexes, but male mutants are able to maintain nearly normal bone mass, presumably by altering glucose distribution.

Osteoblast-specific Cpt2 mutants are sensitized to high-fat diet feeding. We next questioned whether challenging male $\Delta \mathrm{Cpt} 2$ mice with a high-fat diet would result in more severe skeletal and metabolic phenotypes, since high-fat diet feeding and the ensuing accumulation of adipose tissue increases estrogen levels in males (Figure 5A) $(44,45)$. Cohorts of 4 -week-old male control and $\Delta \mathrm{Cpt} 2$ mice were fed a high-fat diet $(60 \%$ of calories from fat) for 8 weeks before analysis. While control and mutant mice exhibited similar increases in body weight (Figure 5B), microCT analysis revealed significant bone loss in both the distal femur and L5 vertebrae of the high-fat diet-fed $\Delta \mathrm{Cpt} 2$ mice (Figure 5, C-G). Indeed, while high-fat diet feeding produced a mild increase in the trabecular bone volume of control mice relative to age-matched chow-fed mice ( $16.3 \%$ in the distal femur, Figure $5 \mathrm{G}$ ), bone volume was reduced by $33.4 \%$ in the femurs of $\Delta \mathrm{Cpt} 2$ mice, which serum analysis suggested was primarily due to a reduction in bone formation (Figure 5, $\mathrm{H}$ and I).

At necropsy, it was clear that the body composition phenotype of $\Delta \mathrm{Cpt} 2$ mice had been reversed by high-fat diet feeding, such that the weights of all major fat pads were increased in the mutants relative to controls (Figure 6A), while visceral organ weights were unaffected (Figure 6B). In line with the increase in fat mass, adipocyte size was increased in both the gonadal and inguinal fat pads (Figure 6, C-E) of the $\Delta \mathrm{Cpt} 2$ mice relative to controls, which is suggestive of a marked increase in adipocyte hypertrophy. Likewise, histological examination of the liver (Figure 6C), as well as the extraction of tissue lipids (Figure $6 \mathrm{~F}$ ), revealed that the $\Delta \mathrm{Cpt} 2$ mice had accumulated more triglyceride within this tissue. Consistent with the phenotype of chow-fed animals, $\Delta \mathrm{Cpt} 2$ mutants remained hyperlipidemic, as serum triglycerides and free fatty acids were elevated relative to controls (Figure 6, G-K). However, whereas chow-fed mutants did not exhibit a change in glucose metabolism (Figure 4L and Supplemental Figure 3), random-fed glucose levels were increased in high-fat diet-fed mutants (Figure 6L). Additionally, the mutant mice were hyperinsulinemic (Figure 6M), performed poorly in glucose tolerance and insulin sensitivity tests (Figure 6, N and O), and exhibited an increase in pancreatic $\beta$ cell islet size (Figure 6, P 

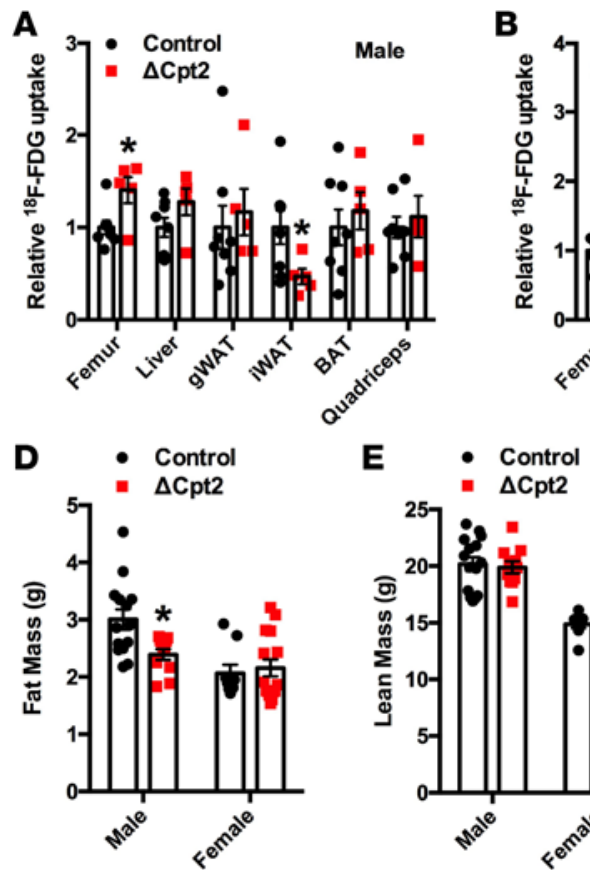

- $\Delta$ Cpt2

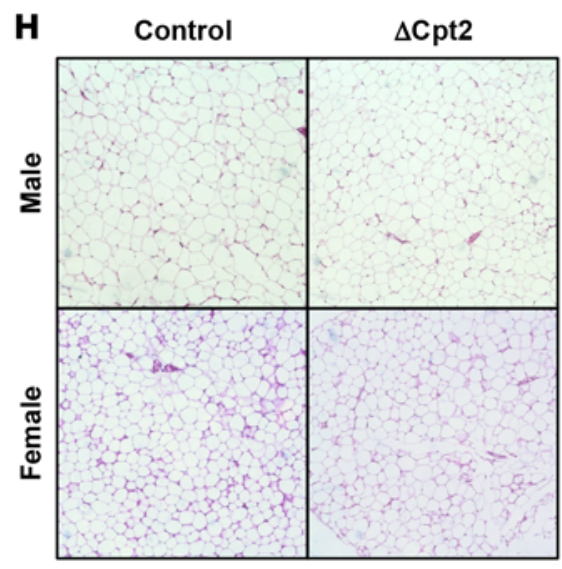

K

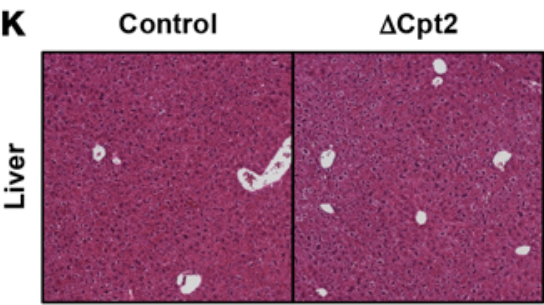

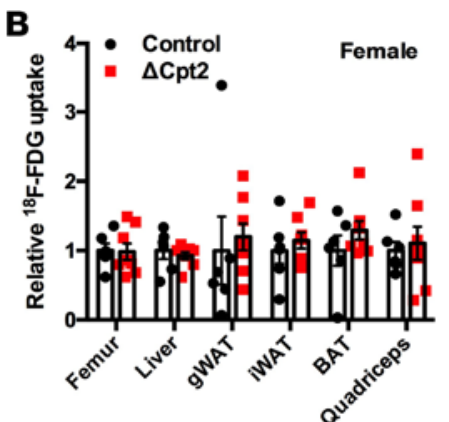
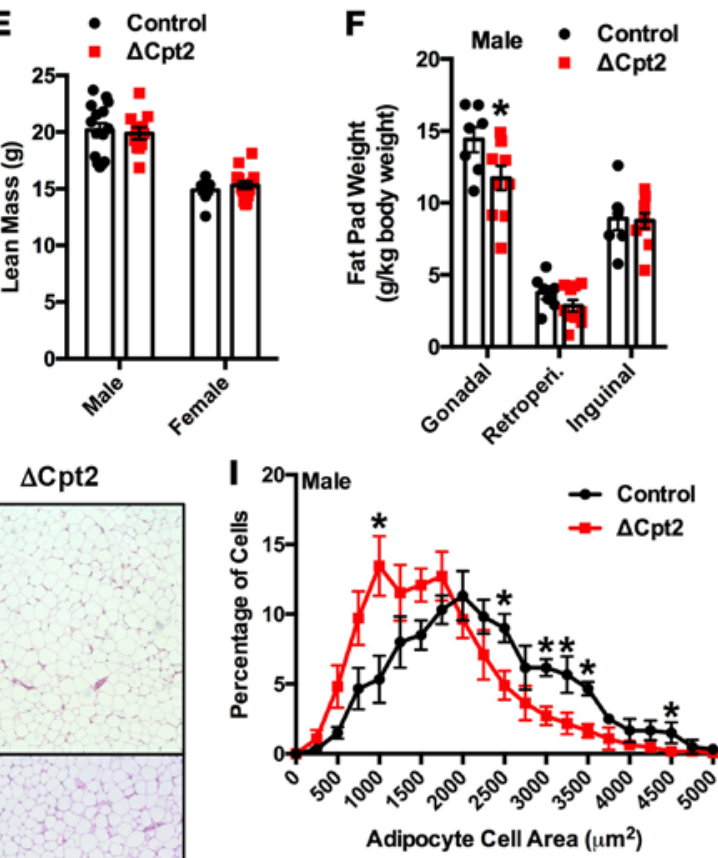

L
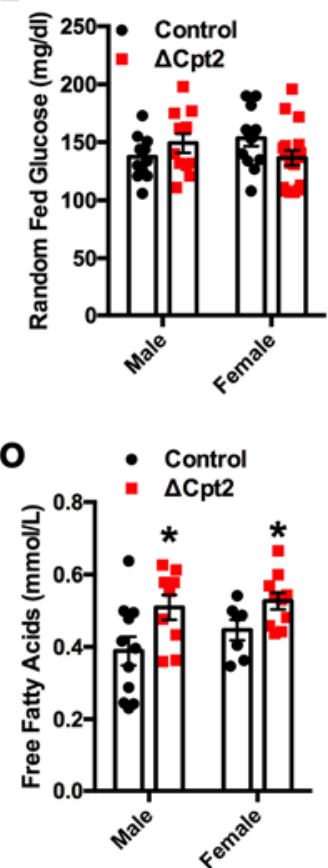

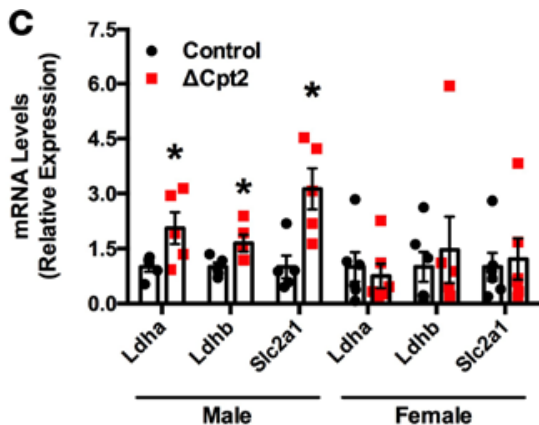

G
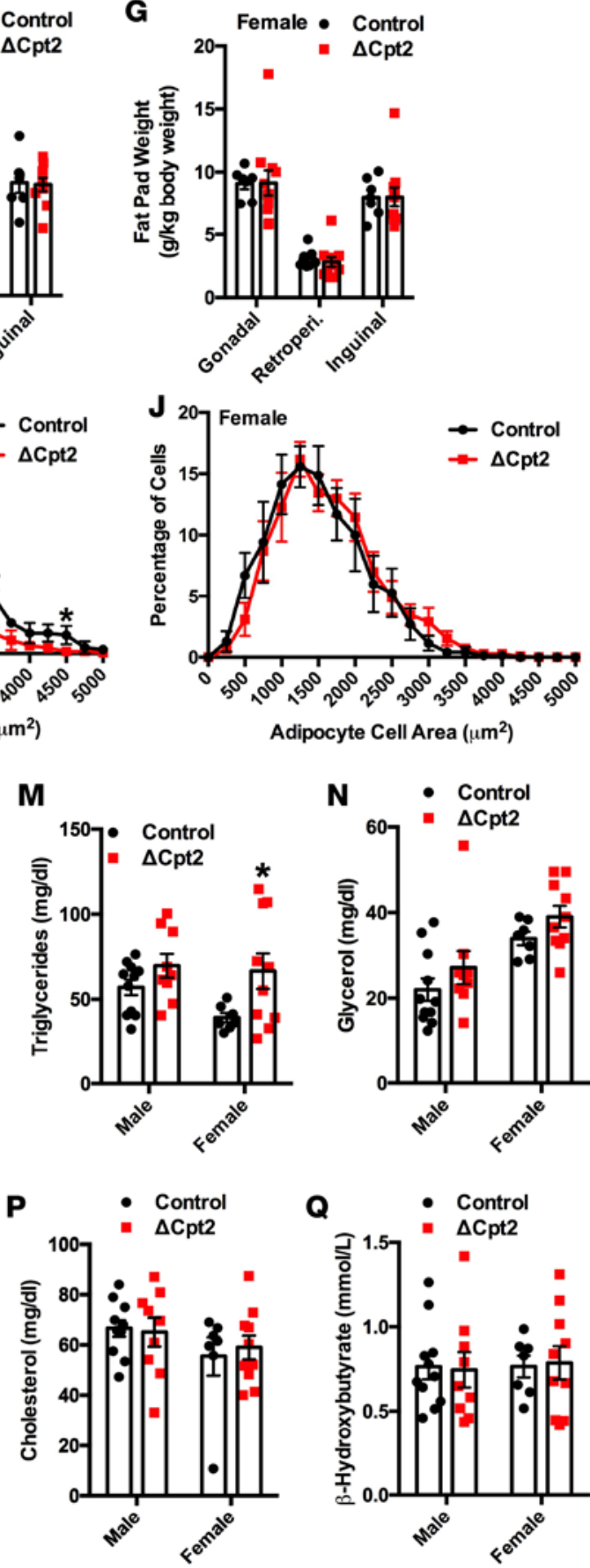

Figure 4. Cpt2 loss of function in osteoblasts alters glucose distribution, lipid homeostasis, and body composition. (A and B) [18 F]-fluorodeoxyglucose (FDG) biodistribution in 12-week-old male (A) and female (B) $\Delta$ Cpt2 mice and their control littermates ( $n=5-8$ mice). (C) qPCR analysis of genes associated with glucose metabolism in the femurs of control and $\Delta$ Cpt2 mice ( $n=5-6$ mice). ( $\mathbf{D}$ and $\mathbf{E})$ qNMR analysis of fat (D) and lean (E) body mass ( $n=9-15$ mice). (F and 
G) Wet weights of adipose depots in 12-week-old male (F) and female (G) mice ( $n=7-11$ mice) . (H) Representative histological images of the gonadal fat pad. Original magnification, 10x (representative of $n=6-11$ mice). (I and J) Frequency distribution of adipocyte size in the gonadal fat pad of male (I) and female (J) mice ( $n=6-11$ mice). (K) Representative histological image of liver in 12 week old male control and $\Delta$ Cpt2 mice. Original magnification, $10 \times$ (representative of $n$ = 6-11 mice). (L) Random-fed blood glucose ( $n=11-17$ mice). (M-Q) Random-fed serum lipid analyses ( $n=7-11$ mice). All data are represented by mean \pm SEM. ${ }^{*} P<0.05$ by unpaired, two-tailed Student's $t$ test.

and $\mathrm{Q}$ ), indicative of the development of insulin resistance. Indeed, the ability of a bolus of insulin to stimulate Akt phosphorylation was reduced in both adipose tissue and bone (Figure 6, R and S), without a significant effect on the circulating levels of undercarboxylated osteocalcin (Figure 6T). Together, these data indicate that the inhibition of fatty acid metabolism in osteoblasts and osteocytes is sufficient to sensitize mice to the detrimental metabolic effects of a high-fat diet feeding and demonstrate the requirement for adequate fatty acid utilization to maintain skeletal mass.

\section{Discussion}

The well-established derangements in bone mass and strength in conditions of malnutrition and disturbed metabolism, as in diabetes mellitus, demonstrate the close association of skeletal metabolism and the acquisition of bone mass with nutrient status and homeostasis (6-11). In this study, we explored the requirement for sufficient fatty acid $\beta$-oxidation during bone acquisition by generating mice that lack $\mathrm{Cpt} 2$ specifically in osteoblasts and osteocytes. Our results demonstrate that fatty acid catabolism is required for normal bone mass accrual and that the impact of impaired $\beta$-oxidation is heavily influenced by sex and diet. To the best of our knowledge, these are the first studies to demonstrate the essential nature of fatty acid oxidation for normal bone acquisition.

As indicated above, the utilization of fatty acids as an energy source by cells of the osteoblast lineage has not been well studied. Early in vitro studies predicted fatty acid oxidation would contribute only a fraction of the energy production of glucose metabolism in osteoblasts (23). However, a more recent study indicated that the skeleton is a site of significant lipoprotein uptake (25), which is in agreement with the levels of radiolabelled bromopalmitate and oleate uptake in selected bones that we report here. The vast majority of studies have instead focused on the detrimental effects of high-fat diet feeding on skeletal homeostasis. In general, these studies have reported a reduction in bone mass secondary to reduced osteoblast activity and increased osteoclastic bone resorption (46-49), as well as reduced sensitivity to anabolic hormones such as parathyroid hormone (PTH) (50).

Our analysis of bone structure revealed defects in trabecular bone structure in both male and female Cpt2 mice, but the phenotype was more severe in female mutants where bone volume was reduced at all time points examined. Histomorphometric studies performed in this sex suggested that the reduction in bone volume was secondary to impairments in mineralization, as the MAR was reduced and parameters of osteoid accumulation were increased. These in vivo data are supported by both the reduced ability of $\Delta \mathrm{Cpt} 2$ osteoblast cultures to form a mineralized matrix and our previous finding of a dramatic increase in fatty acid oxidation as osteoblasts attain a mature phenotype (24). Likewise, these data are consistent with previous studies that reported a linkage between bone mass and the function of CD36 (51) and FFA4 (52), receptors that facilitate fatty acid uptake and are activated by extracellular fatty acids, respectively. However, it is important to keep in mind that these studies were completed in the context of global CD36 or FFA4 deletion and could be confounded by changes in the metabolic activity of other tissues.

The increase in glucose uptake evident in osteoblast cultures deficient for Cpt2 strongly suggests that the defect in osteoblast differentiation is secondary to unmet metabolic demand. Cultures of $\Delta \mathrm{Cpt} 2$ osteoblasts produced more lactate and exhibited an increase in the expression of transcripts encoding Glut1 and lactate dehydrogenase, which are likely a compensatory mechanism intended to maintain ATP levels. However, we cannot completely rule out the possibility that epigenetic mechanisms also contribute to impaired osteoblast differentiation due to Cpt2 deficiency. Much like glucose catabolism, intramitochondrial fatty acid metabolism generates acetyl-CoA that can contribute to chromatin acetylation (53). We plan to explore this possibility in future studies.

The ability to modify fuel selection and increase glucose uptake as a result of impaired long-chain fatty acid metabolism also appears to be at the root of the sexual dimorphism in the skeletal phenotype of Cpt2 mutants. $\left[{ }^{18} \mathrm{~F}\right]-\mathrm{FDG}$ biodistribution studies demonstrated that glucose uptake was increased in male mutants but not in female mutants. Intriguingly, treatment of osteoblast cultures with estrogen, the primary 
A

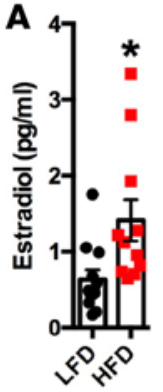

F
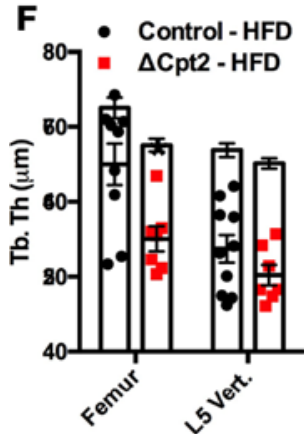

B

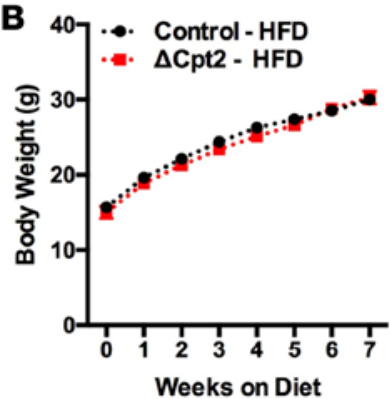

G

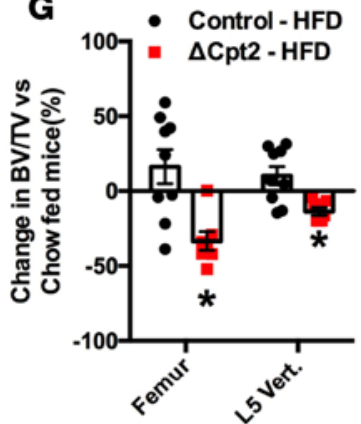

C

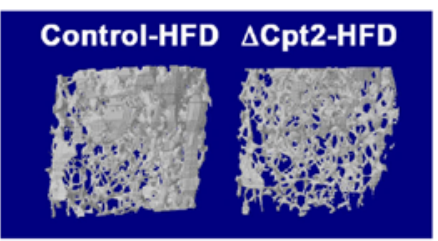

H

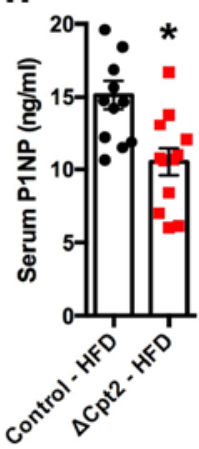

I
D

- Control - HFD

$\Delta$ Cpt2 - HFD
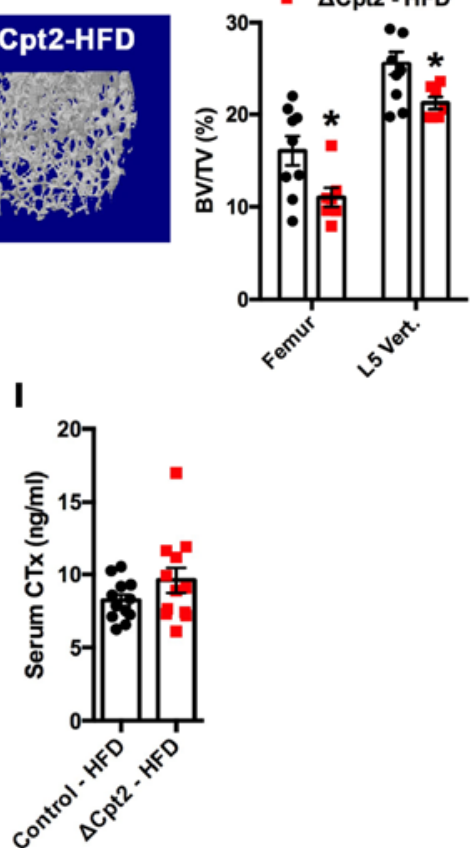

Figure 5. High-fat diet feeding reduces bone volume in male $\Delta \mathbf{C p t 2}$ mice. (A) Serum estradiol levels in male mice fed a low- or high-fat diet ( $n=11-12$ mice). (B-I) Control and $\Delta$ Cpt2 mice were fed a high-fat diet (60\% of calories from fat) from 4-12 weeks of age. (B) Body weight was assessed weekly ( $n$ = 11-13 mice). (C) Representative computer renderings of bone trabecular structure in the distal femur of control and $\Delta$ Cpt2 mice. (D-F) Quantitation of trabecular bone volume per tissue volume (D, BV/TV), trabecular number (E, Tb. N), and trabecular thickness (F, Tb.Th) in the distal femur and L5 vertebrae of high-fat diet-fed mice ( $n=7-10$ mice). (G) Change in trabecular bone volume of high-fat diet-fed control and $\Delta$ Cpt2 mice relative to mice fed a chow diet ( $n=7-10$ mice). (H and I) Serum analysis of the bone turnover markers procollagen type $1 \mathrm{~N}$-terminal propeptide (H, P1NP) and collagen Type I C-terminal telopeptide (I, CTX) ( $n=11-12$ mice). All data are represented by mean \pm SEM. ${ }^{*} P<0.05$ by unpaired, two-tailed Student's $t$ test.

female sex hormone, produced a similar result, wherein glucose uptake and lactate generation were abolished in $\triangle \mathrm{Cpt} 2$ osteoblasts. As a result, cellular ATP levels were reduced and the activation status of AMP$\mathrm{K} \alpha$, which can regulate that activity of Runx2 (21), was increased. Estrogen favors fatty acid metabolism in a number of tissues (39-42) and may therefore prevent the switch to glycolytic metabolism in osteoblasts. This idea is worthy of additional study and will likely require the use of models in which estrogen receptor signaling has been modified in the osteoblast lineage. An impaired ability to modify fuel selection may also explain the precipitous loss of bone mass evident in male Cpt2 mutants fed a high-fat diet over an 8-week period. High-fat diet feeding and the ensuing accumulation of adipose tissue increases the aromatization of testosterone and thereby increases serum estrogen levels even in males (44, 54-56), which could, in turn, prevent glucose utilization in the skeleton of high-fat diet-fed $\Delta \mathrm{Cpt} 2$ mice.

A striking finding of our study was the disturbances in whole-body lipid metabolism and body composition following the loss of Cpt 2 function in osteoblasts and osteocytes. Regardless of diet, both male and female $\Delta \mathrm{Cpt} 2$ mice had increased serum free fatty acid levels, while chow-fed female mutants and high-fat diet-fed male mutants presented with hypertriglyceridemia. The development of dyslipidemia in these models further confirms the idea that cells of the osteoblast lineage are significant utilizers of available fatty acids and is suggestive of a redistribution of energy resources when fatty acid utilization is compromised in the osteoblast. In this regard, the current data are entirely compatible with the conclusions of our previous work (24), which reported an increase in adiposity and the development of dyslipidemia in mice lacking the Wnt-signaling coreceptor Lrp5, specifically in osteoblasts and osteocytes. Based on the reduced expression of enzymatic mediators of fatty acid $\beta$-oxidation in Lrp5-deficient osteoblasts, we hypothesized that Wnt signaling promotes fatty acid utilization in the osteoblasts and that reductions in Wnt signaling result in reduced lipid utilization and its storage in adipose tissue (24). The similarities in the metabolic phenotypes of mice deficient for Lrp5 and Cpt2 in osteoblasts and osteocytes lend credence to this idea.

A redistribution of fatty acids likely explains the increase in fat mass, adipocyte hypertrophy, and hepatic steatosis evident in Cpt2 mutants fed a high-fat diet. Since the levels of undercarboxylated osteocalcin, a 
A
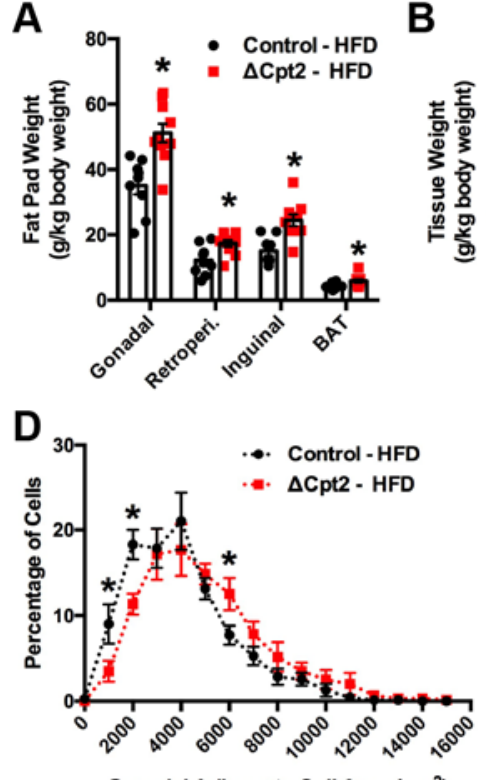

Gonadal Adipocyte Cell Area $\left(\mu \mathrm{m}^{2}\right)$

B

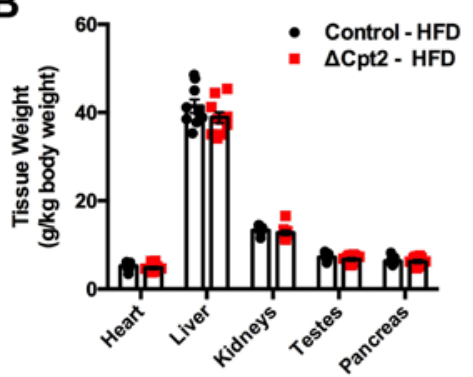

E

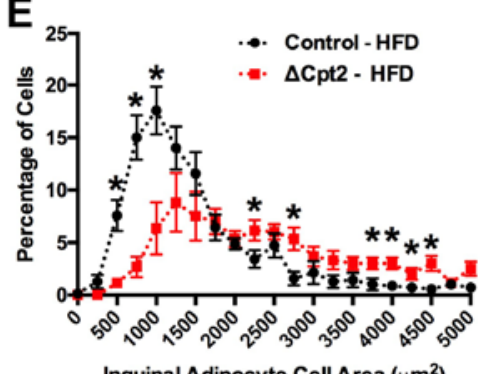

C

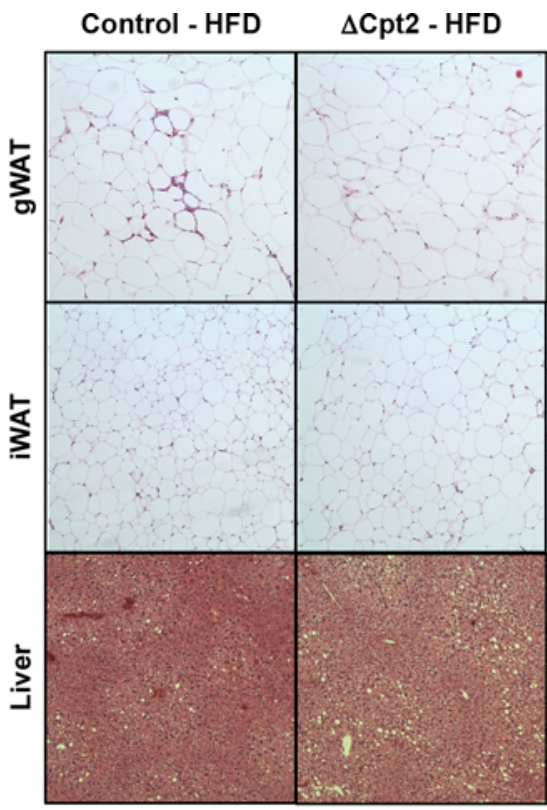

$\mathbf{F}$
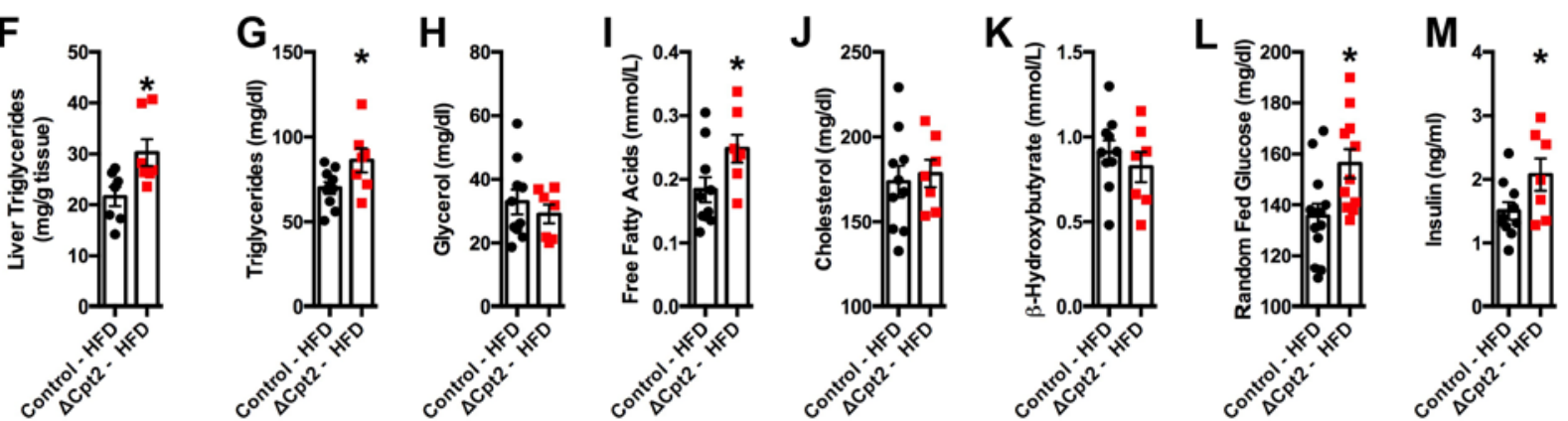

N

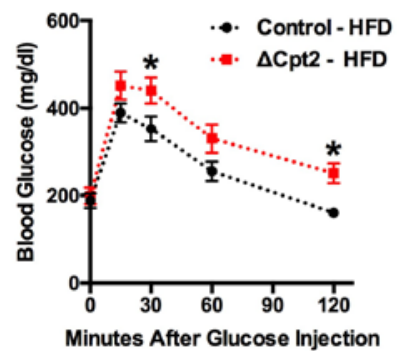

0
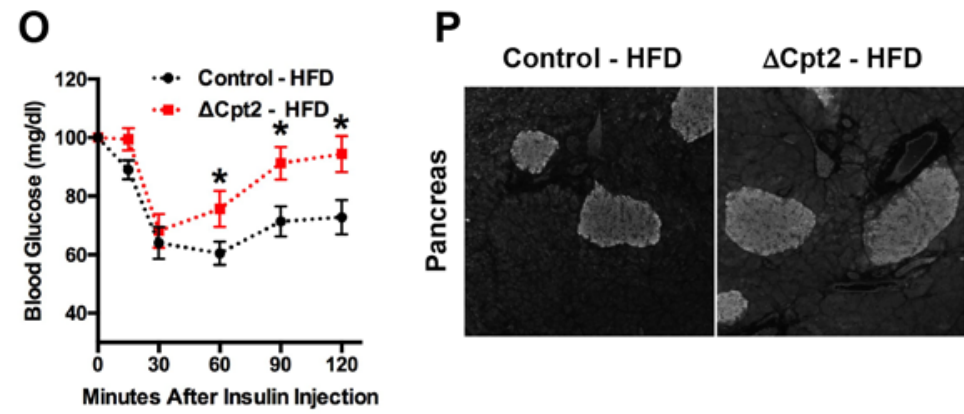

Q

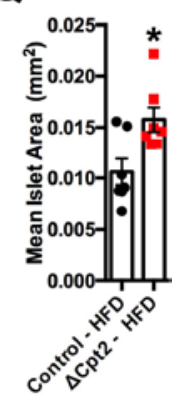

$\mathbf{R}$

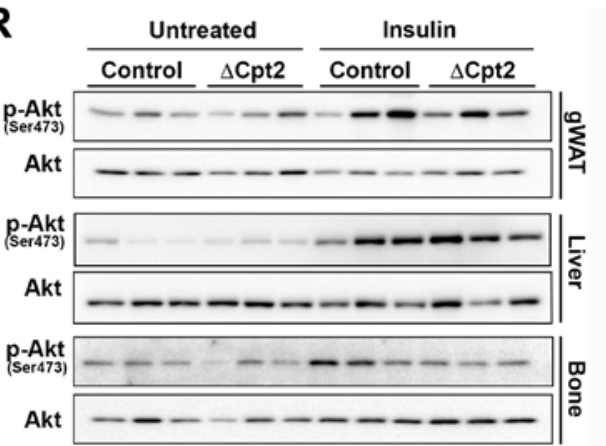

$\mathbf{S}$

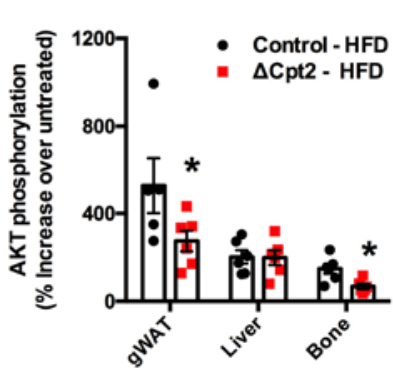

$T$

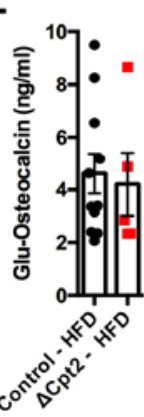

Figure 6. $\Delta$ Cpt2 mice are sensitized to high-fat diet feeding-induced disturbances in metabolism. Control and $\Delta$ Cpt2 mice were fed a high-fat diet (60\% of calories from fat) from 4-12 weeks of age. (A) Adipose depot weights ( $n=8-10$ mice). (B) Wet weights of major organs ( $n=8-10$ mice). (C) Representative histological images of liver, gonadal adipose, and inguinal adipose. Original magnification, 10x (representative of $n=6-7$ mice). ( $D$ and $\mathbf{E}$ ) Frequency distribution of adipocyte size in the gonadal (D) and inguinal (E) fat pads ( $n=6-7$ mice). (F) Liver triglycerides ( $n=7$ mice). (G-K) Random-fed serum lipid 
analyses ( $n=7-10$ mice). (L) Random-fed blood glucose ( $n=11-13$ mice). (M) Random-fed serum insulin ( $n=7-10$ mice). ( $\mathbf{N}$ ) Glucose tolerance testing and (0) insulin tolerance testing ( $n=7-10$ mice). (P) Representative histological images of pancreatic islets after immunostaining for insulin. (Q) Quantification of mean islet area ( $n=7$ mice). (R and $\mathbf{S}$ ) Analysis of Akt phosphorylation in gonadal adipose, liver, and bone after a bolus of insulin ( $n=6$ mice). ( $\mathbf{~})$ Serum Glu-osteocalcin ( $n=5-11$ mice). All data are represented by mean \pm SEM. ${ }^{*} P<0.05$ by unpaired, two-tailed Student's $t$ test.

putative bone-derived regulator of insulin sensitivity, were not affected in $\Delta \mathrm{Cpt} 2$ mice, these phenotypes are expected to contribute to the reduced glucose tolerance and development of insulin resistance in the mutant mice. Given our previous experience with mouse models in which fatty acid utilization by osteoblasts is reduced (24), we were surprised that a similar increase in adiposity was not evident in chow-fed mutants. Instead, quantitative NMR (qNMR) analysis of chow-fed male mutants indicated a reduction in fat mass, which was most evident in the gonadal fat pad. As indicated above, this phenotype is predicted to be the result of altered glucose utilization and a renormalization of glucose storage.

Whether or to what extent enhancing fatty acid oxidation by the osteoblast could be used to increase bone mass or improve serum lipid profiles is currently unknown but is deserving of additional study. The observations that treating in vitro osteoblast cultures with carnitine, a cofactor in the transport of fatty acids into the mitochondria, increases fatty acid oxidation and collagen production (57) - and that carnitine supplementation may improve bone mineral density in ovariectomized rats $(58,59)$ - raises the possibility of an anabolic effect. It is plausible that anabolic therapies designed to improve bone mass might act to increase fatty acid utilization in the skeleton. As an example, parathyroid hormone has recently been demonstrated to increase the metabolic activity of adipose tissue (60). The role of this hormone in osteoblast metabolism has largely been restricted to its affect on glycolysis (19), even though cAMP signaling, a major effector of PTH, promotes fatty acid utilization $(61,62)$.

In summary, our study further confirms the direct link between bone cell metabolism and whole-body metabolism. While it was previously assumed that cells of the osteoblast lineage were primarily glycolytic in nature, it is now clear that fatty acid metabolism is essential to postnatal bone development and homeostasis and that skeletal fatty acid utilization contributes to whole-body lipid homeostasis.

\section{Methods}

Supplemental Methods are available online with this article.

Animal models. The generation of mice in which exon 4 of the mouse Cpt 2 gene is flanked by loxP sequences was previously described $(35,36)$. To generate osteoblast- and osteocyte-specific $\mathrm{KO}, \mathrm{Cpt} 2^{\text {lox } / \mathrm{lox}}$ mice were crossed with Osteocalcin-Cre (Oc-Cre ${ }^{\mathrm{TG} /+}$ ) mice (37). PCR analysis of ear or tail biopsies was used to confirm genotypes. See Supplemental Table 1 for primers used for analysis. All mice were maintained on a C57B1/6 background. Mice were housed on ventilated racks on a 14-hour light/10-hour dark cycle and fed ad libitum with a standard chow diet (Extruded Global Rodent Diet, Harlan Laboratories). For the diet-induced obesity studies, mice were fed a 60\% high-fat diet (D12492, Research Diets) from 4 weeks of age. C57BL/6 mice were obtained from The Jackson Laboratories and acclimated for at least 2 weeks prior to use.

Lipid and glucose biodistribution. For lipid biodistribution studies, $5 \mu \mathrm{Ci}{ }^{3} \mathrm{H}$-bromopalmitate or ${ }^{14} \mathrm{C}$-oleate (PerkinElmer) were administered by gavage in $200 \mu 1$ of olive oil. After a 3-hour uptake period, tissues were collected and weighed, and lipids were extracted by the Folch method (63). Aliquots of the samples were counted for $\left[{ }^{3} \mathrm{H}\right]$ - or $\left[{ }^{14} \mathrm{C}\right]$-labeled lipids using a Beckman Coulter scintillation counter (LS 6000SC, Beckman Coulter). To measure de novo lipogenesis, $10 \mu \mathrm{Ci}{ }^{3} \mathrm{H}$-acetate was administered i.p. 3 hours before tissue collection and processing as above. For all lipid measurements, counts of activity were normalized to the weight of resected tissue. BMIPP was a gift from F. F. Knapp Jr. (Oak Ridge National Laboratory, Oak Ridge, Tennessee, USA) and was labeled with ${ }^{125} \mathrm{I}$ (PerkinElmer) in house. Purity was verified by thin-layer chromatography (TLC) on aluminum-backed silica gel G plate (Merck KGaA) using 80:20:5 petroleum ether/diethyl ether/acetic acid. One hour after retro-orbital administration of BMIPP, tissues were harvested, frozen in optimal cutting media, and sectioned using a Leica 1800 cryostat at a thickness of $10 \mu \mathrm{m}$. Standard sensitivity phosphor-screens (PerkinElmer) were exposed for 10 days at $-20^{\circ} \mathrm{C}$. Digital autoradiograms were acquired using the Cyclone Phosphorimager with the AutoQuant software (Packard).

Clinical grade $\left[{ }^{18} \mathrm{~F}\right]-F D G$ was obtained from the Johns Hopkins PET Center Radio-pharmacy, supplied by PETNET Solutions (Siemens Healthcare). Injectate was assayed using a sodium iodide CRC-15 dose calibrator and a calibration factor of 439 (Capintec). Following a 5-hour fast, mice were injected via the 
retro-orbital sinus with $100 \mu \mathrm{Ci}$ of tracer diluted to a final volume of $150 \mu \mathrm{l}$ in isotonic saline. Following a 2-hour uptake period, under continuous isoflurane anesthesia, animals were sacrificed and tissues were collected. Tissue activity was assessed by $\gamma$ counting, using an energy set $\pm 50 \mathrm{keV}$ from the $511 \mathrm{keV}$ photopeak (Wizard2, PerkinElmer). $\left.{ }^{[18} \mathrm{F}\right]-\mathrm{FDG}$ levels were normalized to the weight of resected tissue and expressed relative to control tissues after calculation of the percent injected dose per gram tissue weight $(\% \mathrm{ID} / \mathrm{g})$.

Skeletal phenotyping. High-resolution images of the mouse femur and L5 vertebra were acquired using a desktop microtomographic imaging system (Skyscan 1172, Bruker) in accordance with the recommendations of the American Society for Bone and Mineral Research (ASBMR) (64). Bones were scanned at $50 \mathrm{keV}$ and $200 \mu \mathrm{A}$ using a $0.5-\mathrm{mm}$ aluminum filter with an isotropic voxel size of $10 \mu \mathrm{m}$. In the femur, trabecular bone parameters were assessed in the $500 \mu \mathrm{m}$ proximal to the growth plate and extending for 2 mm (200 CT slices). Femoral cortical bone structure was assessed in a $500-\mu \mathrm{m}$ region of interest centered on the middiaphysis. In the spine, trabecular bone parameters were assessed between the cranial and caudal growth plates. Dynamic bone formation was assessed at 4 and 12 weeks of age by injection of 2 sequential $25-\mathrm{ml}$ doses of calcein $(0.8 \mathrm{mg} / \mathrm{ml})$. The femur was fixed in ethanol, dehydrated, and embedded in methylmethacrylate. Sections $(3 \mu \mathrm{m})$ were cut with a Microm microtome and stained with Masson-Goldner trichrome stain. The number of osteoblasts and osteoclasts per bone perimeter were measured at standardized sites under the growth plate at a magnification of 200× using a semiautomatic method (Osteoplan II, Kontron). These parameters comply with the guidelines of the nomenclature committee of the ASBMR $(65,66)$. Serum bone turnover markers P1NP and CTx were measured by ELISA (IDS, catalogs AC-33F1 and AC-06F1, respectively).

Culture of primary osteoblasts. Mouse osteoblasts were isolated from the calvaria of 1- to 3-day-old neonates by serial digestion in $1.8 \mathrm{mg} / \mathrm{ml}$ collagenase (Worthington Biochemical). For in vitro deletion of $\mathrm{Cpt} 2$, osteoblasts isolated from $\mathrm{Cpt} 2^{\text {lox } / \mathrm{lox}}$ mice were infected with adenovirus encoding Cre recombinase or GFP (Vector Biolabs). A multiplicity of infection of 100 was used in all experiments, and gene deletion was confirmed by quantitative PCR (qPCR). Osteoblast differentiation was induced by supplementing $\alpha$ MEM containing $10 \%$ serum with $10 \mathrm{mM} \beta$-glycerol phosphate and $50 \mu \mathrm{g} / \mathrm{ml}$ ascorbic acid. Differentiation was confirmed by Alizarin red S staining for mineralization according to standard techniques. In some studies, $10 \mathrm{nM} 17 \beta$-estradiol (Sigma-Aldrich) was added to the culture media

In vitro metabolic studies. Fatty acid oxidation by osteoblast cultures was measured in flasks with stoppers equipped with center wells as previously described (36). Reactions were incubated at $37^{\circ} \mathrm{C}$ in media containing $0.5 \mathrm{mM}$ L-carnitine, $0.2 \% \mathrm{BSA}$, and ${ }^{14} \mathrm{C}$-oleate (PerkinElmer). Expired ${ }^{14} \mathrm{CO}_{2}$ was captured and counted by the addition of $1 \mathrm{~N}$ perchloric acid to the reaction mixture and $1 \mathrm{M} \mathrm{NaOH}$ to the center well containing Whatman filter paper. The acidified reaction mixture was incubated overnight at $4^{\circ} \mathrm{C}$ and centrifuged at $13 \mathrm{~g}$ for 30 minutes before aliquots of the supernatant were counted for ${ }^{14} \mathrm{C}$-labeled acid soluble metabolites. Glucose uptake was assessed as previously described $(67,68)$. Briefly, osteoblast cultures were incubated in MEM ( $1 \mathrm{~g} / 1$ glucose $) / 0.1 \%$ BSA at $37^{\circ} \mathrm{C}$ for 2 hours. The cells were then washed with $\mathrm{KRH}$ buffer (25 mM HEPES-NaOH [pH 7.4], $120 \mathrm{mM} \mathrm{NaCl}, 5 \mathrm{mM} \mathrm{KCl}, 1.2 \mathrm{mM} \mathrm{MgSO}_{4}, 1.3 \mathrm{mM} \mathrm{CaCl}_{2}, 1.3$ $\mathrm{mM} \mathrm{KH}_{2} \mathrm{PO}_{4}$ ) containing no glucose and incubated for an additional 2 hours. Cells were incubated in KRH containing 2-deoxy-D- $\left[{ }^{3} \mathrm{H}\right]$-glucose for 5 minutes. An additional group was treated with cytochalasin B $(10 \mu \mathrm{M})$ to assess nonspecific uptake. Glutamine uptake was assessed via the same method. Uptake of ${ }^{3} \mathrm{H}$-Glucose and ${ }^{3} \mathrm{H}$-Glutamine were detected using a Beckman scintillation counter and normalized to protein content. Cellular lactate (Sigma-Aldrich) and ATP levels (Roche Diagnostics) were measured via commercially available kits (Sigma-Aldrich, MAK064; Roche ATP Bioluminescence Assay HSII, 11699709001). Oxygen consumption and extracellular acidification rate were measured using the Seahorse XFe96 extracellular flux analyzer (Seahorse Bioscience). Cells $(20,000)$ were seeded in 96 -well microplates and then differentiated in the presence or absence of $\mathrm{E} 2$ for 7 days. Cells were incubated in minimal media (5 mM HEPES [pH 7.4], $2.5 \mathrm{mM}$ glucose, $111 \mathrm{mM} \mathrm{NaCl}, 4.7 \mathrm{mM} \mathrm{KCl}, 1.25 \mathrm{mM} \mathrm{CaCl}, 2 \mathrm{mM} \mathrm{MgSO}{ }_{4}$, $1.2 \mathrm{mM} \mathrm{Na}_{2} \mathrm{HPO}_{4}, 0.5 \mathrm{mM}$ L-carnitine, and $34 \mu \mathrm{M} \mathrm{BSA}$ ) supplemented with $200 \mu \mathrm{M}$ oleate or $2.5 \mathrm{mM}$ glucose for 1 hour before assessing cellular respiration. Results were normalized to cell number determined by CyQUANT (Invitrogen) staining of cellular nucleic acids. Antibodies specific for AMPK isoforms were obtained from Cell Signaling Technology (p-AMPK $\alpha$, catalog 2534; AMPK $\alpha$, catalog 5831; p-AMPK $\beta 1$, catalog 4186; AMPK $\beta 1 / 2$, catalog 4150)

Gene expression studies. Total RNA was extracted from cell cultures or mouse tissue using TRIzol (Life Technologies). Reverse transcriptase reactions were carried out using $1 \mu \mathrm{g}$ of RNA and the iScript cDNA 
Synthesis system (Bio-Rad). qPCR was carried out using iQ Sybr Green Supermix (Bio-Rad) using primer sequences obtained from PrimerBank (http://pga.mgh.harvard.edu/primerbank/index.html). Sequences are available in Supplemental Table 1. Reactions were normalized to endogenous $18 \mathrm{~S}$ reference transcripts.

Metabolic studies and bioassays. Whole-body fat and lean body mass were assessed by qNMR (Echo MRI). Plasma triglycerides (Sigma-Aldrich), $\beta$-hydroxybutyrate (Sigma-Aldrich), cholesterol (BioAssay Systems), free fatty acids (Sigma-Aldrich), and glycerol (Sigma-Aldrich) were measured colorimetrically in plasma collected 3 hours after the initiation of the light cycle. Glucose levels were measured using a hand-held OneTouch Ultra glucose monitor. For glucose tolerance testing, glucose ( $2 \mathrm{~g} / \mathrm{kg} \mathrm{BW}$ or $1 \mathrm{~g} / \mathrm{kg} \mathrm{BW}$ for high-fat diet mice) was injected i.p. after a 6-hour fast. For insulin-tolerance testing, mice were fasted for 4 hours and then injected i.p. with insulin $(0.4 \mathrm{U} / \mathrm{kg} \mathrm{BW}$ or $1 \mathrm{U} / \mathrm{kg} \mathrm{BW}$ for HFD mice). Total Akt (Cell Signaling Technology, 9272) and phosphorylated Akt (Cell Signaling Technology, 9271) levels in tissues were assessed by Western blotting using lysates collected immediately prior and 5 minutes after the administration of insulin. Serum levels of insulin (Alpco, 80-INSMSU-E01), estradiol (Calbiotech, ES180S-100), and Glu-osteocalcin (Takara Clonetech, MK118) were assessed by ELISA. Pancreata were fixed and stained, and islet morphometry was assessed as previously described (69). Liver and adipose tissues were fixed in paraformaldehyde and processed for immunostaining or $\mathrm{H} \& \mathrm{E}$ staining according to standard techniques. Antibodies specific for AKT were obtained from Cell Signaling Technology (p-AKT, catalog 4060; AKT, catalog 4685).

Statistics. All results are presented as means \pm SEM. Statistical analyses were performed using unpaired, two-tailed Student's $t$ or ANOVA tests followed by post hoc tests. A $P$ value less that 0.05 was considered significant. In all figures, ${ }^{*} P \leq 0.05$.

Study approval. The IACUC of the Johns Hopkins University approved all procedures involving mice.

\section{Author contributions}

Conceptualization contributed by RCR, MJW, and TLC; investigation contributed by SPK, ZL, MLZ, JLF, CEB, PK, KAR, BCG, SS, JEP, EEK, MCF, DLJT, RCR; writing of original draft contributed by RCR, MJW, and TLC; review and editing of manuscript contributed by RCR, MJW, EEK, and TLC; funding acquisition contributed by RCR, MJW, and TLC; supervision contributed by RCR.

\section{Acknowledgments}

We are indebted to Nadine Forbes-McBean in the Johns Hopkins University Phenotyping Core. This work was supported by NIH grants DK099134 to RCR, NS072241 to MJW, and DK090166 to EEK and a Merit Review grant (BX001234) from the Biomedical Laboratory Research and Development Service of the Veterans Affairs Office of Research and Development to TLC. TLC is also the recipient of a Research Career Scientist Award from the Department of Veterans Affairs. Support was also provided by the JHU-UMD Diabetes Research Center (DK079637).

Address correspondence to: Ryan C. Riddle, Department of Orthopaedic Surgery, Johns Hopkins University School of Medicine, 1721 E. Madison Street, Baltimore, Maryland 21205, USA. Phone: 410.502.6412; Email: rriddle1@jhmi.edu.

1. Zhang Q, Riddle RC, Clemens TL. Bone and the regulation of global energy balance. J Intern Med. 2015;277(6):681-689.

2. Fulzele $\mathrm{K}$, et al. Insulin receptor signaling in osteoblasts regulates postnatal bone acquisition and body composition. Cell. 2010;142(2):309-319.

3. Ducy P, et al. Leptin inhibits bone formation through a hypothalamic relay: a central control of bone mass. Cell. 2000;100(2):197-207.

4. Ferron M, et al. Insulin signaling in osteoblasts integrates bone remodeling and energy metabolism. Cell. 2010;142(2):296-308

5. Kajimura D, et al. Adiponectin regulates bone mass via opposite central and peripheral mechanisms through FoxO1. Cell Metab. 2013;17(6):901-915.

6. Boyer PM, et al. Bone status in an animal model of chronic sub-optimal nutrition: a morphometric, densitometric and mechanical study. Br J Nutr. 2005;93(5):663-669.

7. Bolton JG, Patel S, Lacey JH, White S. A prospective study of changes in bone turnover and bone density associated with regaining weight in women with anorexia nervosa. Osteoporos Int. 2005;16(12):1955-1962.

8. Nussbaum M, Baird D, Sonnenblick M, Cowan K, Shenker IR. Short stature in anorexia nervosa patients. J Adolesc Health Care. 1985;6(6):453-455.

9. Kemink SA, Hermus AR, Swinkels LM, Lutterman JA, Smals AG. Osteopenia in insulin-dependent diabetes mellitus; preva- 
lence and aspects of pathophysiology. J Endocrinol Invest. 2000;23(5):295-303.

10. Vestergaard P. Discrepancies in bone mineral density and fracture risk in patients with type 1 and type 2 diabetes--a meta-analysis. Osteoporos Int. 2007;18(4):427-444.

11. Janghorbani M, Van Dam RM, Willett WC, Hu FB. Systematic review of type 1 and type 2 diabetes mellitus and risk of fracture. Am J Epidemiol. 2007;166(5):495-505.

12. Cohn DV, Forscher BK. Aerobic metabolism of glucose by bone. J Biol Chem. 1962;237:615-618.

13. Flanagan B, Nichols G. METABOLIC STUDIES OF BONE IN VITRO. V. GLUCOSE METABOLISM AND COLLAGEN BIOSYNTHESIS. J Biol Chem. 1964;239:1261-1265.

14. Neuman WF, Neuman MW, Brommage R. Aerobic glycolysis in bone: lactate production and gradients in calvaria. Am J Physiol. 1978;234(1):C41-C50.

15. Thomas DM, et al. Modulation of glucose transport by parathyroid hormone and insulin in UMR 106-01, a clonal rat osteogenic sarcoma cell line. J Mol Endocrinol. 1995;14(2):263-275.

16. Vaes G, Nichols G. Metabolic studies of bone in vitro. III. Citric acid metabolism and bone mineral solubility. Effects of parathyroid hormone and estradiol. J Biol Chem. 1961;236:3323-3329.

17. Zoch ML, Abou DS, Clemens TL, Thorek DL, Riddle RC. In vivo radiometric analysis of glucose uptake and distribution in mouse bone. Bone Res. 2016;4:16004.

18. Esen E, Chen J, Karner CM, Okunade AL, Patterson BW, Long F. WNT-LRP5 signaling induces Warburg effect through mTORC2 activation during osteoblast differentiation. Cell Metab. 2013;17(5):745-755.

19. Esen E, Lee SY, Wice BM, Long F. PTH Promotes Bone Anabolism by Stimulating Aerobic Glycolysis via IGF Signaling J Bone Miner Res. 2015;30(11):2137.

20. Guntur AR, Le PT, Farber CR, Rosen CJ. Bioenergetics during calvarial osteoblast differentiation reflect strain differences in bone mass. Endocrinology. 2014;155(5):1589-1595.

21. Wei J, et al. Glucose Uptake and Runx2 Synergize to Orchestrate Osteoblast Differentiation and Bone Formation. Cell. 2015;161(7):1576-1591.

22. Li Z, et al. Glucose Transporter-4 Facilitates Insulin-Stimulated Glucose Uptake in Osteoblasts. Endocrinology. 2016;157(11):4094-4103.

23. Adamek G, Felix R, Guenther HL, Fleisch H. Fatty acid oxidation in bone tissue and bone cells in culture. Characterization and hormonal influences. Biochem J. 1987;248(1):129-137.

24. Frey JL, et al. Wnt-Lrp5 signaling regulates fatty acid metabolism in the osteoblast. Mol Cell Biol. 2015;35(11):1979-1991.

25. Niemeier A, et al. Uptake of postprandial lipoproteins into bone in vivo: impact on osteoblast function. Bone. 2008;43(2):230-237.

26. Catherwood BD, Addison J, Chapman G, Contreras S, Lorang M. Growth of rat osteoblast-like cells in a lipid-enriched culture medium and regulation of function by parathyroid hormone and 1,25-dihydroxyvitamin D. J Bone Miner Res. 1988;3(4):431-438.

27. Britton CH, Schultz RA, Zhang B, Esser V, Foster DW, McGarry JD. Human liver mitochondrial carnitine palmitoyltransferase I: characterization of its cDNA and chromosomal localization and partial analysis of the gene. Proc Natl Acad Sci USA. 1995;92(6):1984-1988.

28. Yamazaki N, Shinohara Y, Shima A, Yamanaka Y, Terada H. Isolation and characterization of cDNA and genomic clones encoding human muscle type carnitine palmitoyltransferase I. Biochim Biophys Acta. 1996;1307(2):157-161.

29. Price N, et al. A novel brain-expressed protein related to carnitine palmitoyltransferase I. Genomics. 2002;80(4):433-442.

30. Wolfgang MJ, Lane MD. Control of energy homeostasis: role of enzymes and intermediates of fatty acid metabolism in the central nervous system. Annu Rev Nutr. 2006;26:23-44.

31. Wolfgang MJ, et al. The brain-specific carnitine palmitoyltransferase-1c regulates energy homeostasis. Proc Natl Acad Sci USA. 2006;103(19):7282-7287.

32. Haynie KR, Vandanmagsar B, Wicks SE, Zhang J, Mynatt RL. Inhibition of carnitine palymitoyltransferase1b induces cardiac hypertrophy and mortality in mice. Diabetes Obes Metab. 2014;16(8):757-760

33. Knapp FF, Goodman MM, Callahan AP, Kirsch G. Radioiodinated 15-(p-iodophenyl)-3,3-dimethylpentadecanoic acid: a useful new agent to evaluate myocardial fatty acid uptake. J Nucl Med. 1986;27(4):521-531.

34. Shearer J, Coenen KR, Pencek RR, Swift LL, Wasserman DH, Rottman JN. Long chain fatty acid uptake in vivo: comparison of [125I]-BMIPP and [3H]-bromopalmitate. Lipids. 2008;43(8):703-711.

35. Lee J, Choi J, Scafidi S, Wolfgang MJ. Hepatic Fatty Acid Oxidation Restrains Systemic Catabolism during Starvation. Cell Rep. 2016;16(1):201-212.

36. Lee J, Ellis JM, Wolfgang MJ. Adipose fatty acid oxidation is required for thermogenesis and potentiates oxidative stress-induced inflammation. Cell Rep. 2015;10(2):266-279.

37. Zhang M, et al. Osteoblast-specific knockout of the insulin-like growth factor (IGF) receptor gene reveals an essential role of IGF signaling in bone matrix mineralization. J Biol Chem. 2002;277(46):44005-44012.

38. Zimmermann R, et al. Fat mobilization in adipose tissue is promoted by adipose triglyceride lipase. Science. 2004;306(5700):1383-1386.

39. Campbell SE, Febbraio MA. Effect of ovarian hormones on mitochondrial enzyme activity in the fat oxidation pathway of skeletal muscle. Am J Physiol Endocrinol Metab. 2001;281(4):E803-E808.

40. Hatta H, Atomi Y, Shinohara S, Yamamoto Y, Yamada S. The effects of ovarian hormones on glucose and fatty acid oxidation during exercise in female ovariectomized rats. Horm Metab Res. 1988;20(10):609-611.

41. Herrero P, et al. Impact of hormone replacement on myocardial fatty acid metabolism: potential role of estrogen. $J$ Nucl Cardiol. 2005;12(5):574-581

42. Kendrick ZV, Ellis GS. Effect of estradiol on tissue glycogen metabolism and lipid availability in exercised male rats. $J$ Appl Physiol. 1991;71(5):1694-1699.

43. Karner CM, Esen E, Okunade AL, Patterson BW, Long F. Increased glutamine catabolism mediates bone anabolism in response to WNT signaling. J Clin Invest. 2015;125(2):551-562.

44. Fan Y, et al. Diet-induced obesity in male C57BL/6 mice decreases fertility as a consequence of disrupted blood-testis barrier PLoS One. 2015;10(4):e0120775. 
45. Schneider G, Kirschner MA, Berkowitz R, Ertel NH. Increased estrogen production in obese men. J Clin Endocrinol Metab. 1979;48(4):633-638.

46. Chen X, et al. Reduced femoral bone mass in both diet-induced and genetic hyperlipidemia mice. Bone. 2016;93:104-112.

47. Lecka-Czernik B, Stechschulte LA, Czernik PJ, Dowling AR. High bone mass in adult mice with diet-induced obesity results from a combination of initial increase in bone mass followed by attenuation in bone formation; implications for high bone mass and decreased bone quality in obesity. Mol Cell Endocrinol. 2015;410:35-41.

48. Shu L, et al. High-fat diet causes bone loss in young mice by promoting osteoclastogenesis through alteration of the bone marrow environment. Calcif Tissue Int. 2015;96(4):313-323.

49. Lu XM, Zhao H, Wang EH. A high-fat diet induces obesity and impairs bone acquisition in young male mice. Mol Med Rep. 2013;7(4):1203-1208.

50. Huang MS, et al. Hyperlipidemia impairs osteoanabolic effects of PTH. J Bone Miner Res. 2008;23(10):1672-1679.

51. Kevorkova O, Martineau C, Martin-Falstrault L, Sanchez-Dardon J, Brissette L, Moreau R. Low-bone-mass phenotype of deficient mice for the cluster of differentiation 36 (CD36). PLOS ONE. 2013;8(10):e77701.

52. Ahn SH, et al. Free Fatty Acid Receptor 4 (GPR120) Stimulates Bone Formation and Suppresses Bone Resorption in the Presence of Elevated n-3 Fatty Acid Levels. Endocrinology. 2016;157(7):2621-2635.

53. McDonnell E, et al. Lipids Reprogram Metabolism to Become a Major Carbon Source for Histone Acetylation. Cell Rep. 2016;17(6):1463-1472.

54. Cao JJ, Gregoire BR. A high-fat diet increases body weight and circulating estradiol concentrations but does not improve bone structural properties in ovariectomized mice. Nutr Res. 2016;36(4):320-327.

55. Nimrod A, Ryan KJ. Aromatization of androgens by human abdominal and breast fat tissue. J Clin Endocrinol Metab. 1975;40(3):367-372.

56. Longcope C, Pratt JH, Schneider SH, Fineberg SE. Aromatization of androgens by muscle and adipose tissue in vivo. J Clin Endocrinol Metab. 1978;46(1):146-152.

57. Chiu KM, Keller ET, Crenshaw TD, Gravenstein S. Carnitine and dehydroepiandrosterone sulfate induce protein synthesis in porcine primary osteoblast-like cells. Calcif Tissue Int. 1999;64(6):527-533.

58. Hooshmand S, Balakrishnan A, Clark RM, Owen KQ, Koo SI, Arjmandi BH. Dietary 1-carnitine supplementation improves bone mineral density by suppressing bone turnover in aged ovariectomized rats. Phytomedicine. 2008;15(8):595-601.

59. Orsal E, et al. The role of carnitine on ovariectomy and inflammation-induced osteoporosis in rats. Exp Biol Med (Maywood). 2013;238(12):1406-1412.

60. Kir S, et al. PTH/PTHrP Receptor Mediates Cachexia in Models of Kidney Failure and Cancer. Cell Metab. 2016;23(2):315-323.

61. Louet JF, Hayhurst G, Gonzalez FJ, Girard J, Decaux JF. The coactivator PGC-1 is involved in the regulation of the liver carnitine palmitoyltransferase I gene expression by cAMP in combination with HNF4 alpha and cAMP-response element-binding protein (CREB). J Biol Chem. 2002;277(41):37991-38000.

62. Luiken JJ, et al. Effects of cAMP modulators on long-chain fatty-acid uptake and utilization by electrically stimulated rat cardiac myocytes. Biochem J. 2002;367(Pt 3):881-887.

63. Folch J, Lees M, Sloane Stanley GH. A simple method for the isolation and purification of total lipides from animal tissues. J Biol Chem. 1957;226(1):497-509.

64. Bouxsein ML, Boyd SK, Christiansen BA, Guldberg RE, Jepsen KJ, Müller R. Guidelines for assessment of bone microstructure in rodents using micro-computed tomography. J Bone Miner Res. 2010;25(7):1468-1486.

65. Parfitt AM, et al. Bone histomorphometry: standardization of nomenclature, symbols, and units. Report of the ASBMR Histomorphometry Nomenclature Committee. J Bone Miner Res. 1987;2(6):595-610.

66. Dempster DW, et al. Standardized nomenclature, symbols, and units for bone histomorphometry: a 2012 update of the report of the ASBMR Histomorphometry Nomenclature Committee. J Bone Miner Res. 2013;28(1):2-17.

67. Kanda H, et al. Adipocytes from Munc18c-null mice show increased sensitivity to insulin-stimulated GLUT4 externalization. J Clin Invest. 2005;115(2):291-301.

68. Wei Z, et al. C1q/TNF-related protein-12 (CTRP12), a novel adipokine that improves insulin sensitivity and glycemic control in mouse models of obesity and diabetes. J Biol Chem. 2012;287(13):10301-10315.

69. Hussain MA, et al. Increased pancreatic beta-cell proliferation mediated by CREB binding protein gene activation. $\mathrm{Mol}$ Cell Biol. 2006;26(20):7747-7759. 